\title{
Geometric Models for Quasicrystals II. Local Rules Under Isometries
}

\author{
J. C. Lagarias
}

AT\&T Labs, 180 Park Avenue, Florham Park, NJ 07932-0971, USA

jcl@research.att.com

\begin{abstract}
The atomic structures of quasicrystalline materials exhibit long range order under translations. It is believed that such materials have atomic structures which approximately obey local rules restricting the location of nearby atoms. These local constraints are typically invariant under rotations, and it is of interest to establish conditions under which such local rules can nevertheless enforce order under translations in any structure that satisfies them.

A set of local rules $\mathcal{L}$ in $\mathbb{R}^{n}$ is a finite collection of discrete sets $\left\{Y_{i}\right\}$ containing $\mathbf{0}$, each of which is contained in the ball of radius $\rho$ around $\mathbf{0}$ in $\mathbb{R}^{n}$. A set $X$ satisfies the local rules $\mathcal{L}$ under isometries if the $\rho$-neighborhood of each $\mathbf{x} \in X$ is isometric to an element of $\mathcal{L}$. This paper gives sufficient conditions on a set of local rules $\mathcal{L}$ such that if $X$ satisfies $\mathcal{L}$ under isometries, then $X$ has a weak long-range order under translations, in the sense that $X$ is a Delone set of finite type. A set $X$ is a Delone set of finite type if it is a Delone set whose interpoint distance set $X-X$ is a discrete closed set. We show for each minimal Delone set of finite type $X$ that there exists a set of local rules $\mathcal{L}$ such that $X$ satisfies $\mathcal{L}$ under isometries and all other $Y$ that satisfy $\mathcal{L}$ under isometries are Delone sets of finite type.

A set of perfect local rules (under isometries or under translations, respectively) is a set of local rules $\mathcal{L}$ such that all structures $X$ that satisfy $\mathcal{L}$ are in the same local isomorphism class (under isometries or under translations, respectively). If a Delone set of finite type has a set of perfect local rules under translations, then it has a set of perfect local rules under isometries, and conversely.
\end{abstract}

\section{Introduction}

This paper is motivated by the goal of describing the structure of crystals and quasicrystals in terms of "local" conditions, see [50]. The atomic structures of crystalline and quasicrystalline materials exhibit strong long-range order under translations, in the sense of 
having X-ray diffraction patterns containing sharp spots ("Bragg peaks"). The physical mechanisms leading to the formation and stability of such materials are apparantly well described by Gibbs-type statistical mechanics models. These models describe equilibrium structures as arising in a competition between minimum energy and maximum entropy. However, no mathematical derivation determining the possible structures that may occur has been given. ${ }^{1}$ It is empirically observed that the arrangements of atoms in many materials appear to favor special local configurations, which can be called "clusters." This motivates the purely geometric problem of studying those discrete sets $X$ such that the $\rho$-neighborhood of each $\mathbf{x} \in X$ is isometric to one of a finite list $\mathcal{L}$ of $\rho$-neighborhoods of $\mathbf{0}$. We call a set $\mathcal{L}$ used this way a set of local rules under isometries. The object of this paper is to show that a wide variety of structures that possess a weak form of long-range order under translations can arise as a forced consequence of "local rules" which themselves are invariant under the larger group of isometries.

There are many special structures for which such "local rules" have previously been obtained. A set $X$ in $\mathbb{R}^{n}$ is an ideal crystal if it consists of a finite number of translates of a full rank lattice, see [56]. In 1976 Delone et al. [14] showed that local rules under isometries existed for all ideal crystalline structures whose symmetry group is transitive on the set of all points ("regular point system"). A similar result was established for all ideal crystals in Dolbilin et al. [15]. There are now many results giving local rules under isometries characterizing various special classes of aperiodic structures with crystallographically forbidden symmetries, e.g., tilings with 5-, 8-, 10-, and 12-fold rotational symmetries in their diffraction spectrum. These structures typically have perfect local rules, in the sense that all structures that satisfy the local rules are locally isomorphic under translations. They also have strong long-range order under translations which is manifested as a well-defined diffraction pattern, which usually includes some discrete spectrum. For such results, see the survey of Ingersent [29].

The results obtained in this paper apply to a general class of structures $X$ which possess a weak long-range translational order without necessarily having a well-defined diffraction measure. This weak long-range translational order is that the additive group $[X]$ generated by the elements of $X$ is finitely generated, i.e., $[X]$ is a quasilattice in the terminology of Le et al. [39] and Mermin [44]. In the cases where our results apply, the local rules obtained enforce weak long-range translational order on all structures that satisfy them, but these local rules may be imperfect in the sense that the structures satisfying them may not all be locally isomorphic.

We formulate basic definitions. Delone sets are a mathematical idealization of a "solid-state" structure.

Definition 1.1. A Delone set or $(r, R)$-set is any set in $\mathbb{R}^{n}$ having the following two properties:

(i) Uniformly Discrete. There is an $r>0$ such that each open ball of radius $r$ contains at most one element of $X$.

\footnotetext{
${ }^{1}$ It is still an open problem to prove rigorously that a crystalline structure can arise as a minimal energy arrangement of atoms under electromagnetic forces. See [49], [50], and Problem 11 of [58].
} 
(ii) Relatively Dense. There is an $R>0$ such that each closed ball of radius $R$ contains at least one point of $X$.

In part I we introduced a large class of discrete sets in $\mathbb{R}^{n}$ possessing a weak long-range translational order which we called Delone sets of finite type. This class of sets apparently includes nearly all proposed models for the atomic arrangements of quasicrystalline materials, as well as other sets, see [33], [34], [35], [40], [41], [45], and [46].

Definition 1.2. A Delone set of finite type is any Delone set $X$ such that $X-X$ is a discrete closed set, i.e., the intersection of $X-X$ with any closed ball is a finite set.

In part I we showed that a set $X$ is a Delone set of finite type if and only if, for each $T>0$, it has only finitely many different $T$-neighborhoods of a point under translations. Here the T-neighborhood of a point $\mathbf{x} \in X$ is

$$
\mathcal{P}_{X}(\mathbf{x} ; T):=X \cap B(\mathbf{x} ; T),
$$

where $B(\mathbf{x} ; T)$ is the closed ball of radius $T$ centered at $\mathbf{x}$. A centered $T$-patch $\mathcal{P}=$ $\mathcal{P}_{X}(\mathbf{x} ; T)-\mathbf{x}$ is any translate of a $T$-neighborhood to the origin. Part I showed that Delone sets of finite type are characterized by local rules under translations, i.e., a set $X$ is a Delone set of finite type if and only if the set $\mathcal{L}$ of centered $2 R$-patches of $X$ is finite; any set $Y$ that satisfies $\mathcal{L}$ under translations is a Delone set of finite type. An important property of Delone sets of finite type $X$ is that they are finitely generated, i.e., the group of translations $[X]$ spanned by the vectors in $X$ is finitely generated. Thus $[X]$ is a quasilattice; that is, a finitely generated group of translations in $\mathbb{R}^{n}$ that spans $\mathbb{R}^{n}$ as an $\mathbb{R}$-vector space, see [39]. By picking a basis $\left[\mathbf{v}_{1}, \ldots, \mathbf{v}_{s}\right]$ of this $\mathbb{Z}$-module $[X]$ we can describe elements of $X$ using $s$ integer coordinates, and we may regard $X$ as a linear projection of a subset $\tilde{X}$ of the lattice $\mathbb{Z}^{s}$ in $\mathbb{R}^{s}$. In particular, such sets $X$ can always be described using a finite number of "internal dimensions."

The basic question that this paper addresses is: For which Delone sets of finite type $X$ do there exist local rules $\mathcal{L}$ under isometries such that $X$ satisfies $\mathcal{L}$ and any set $Y$ that satisfies $\mathcal{L}$ is also a Delone set of finite type? Our main result is that the class of sets $X$ for which such local rules under isometries exist includes all Delone sets of finite type that are minimal. A Delone set of finite type $X$ is minimal if it is repetitive (under translations), i.e., there is a function $M_{X}(T)$ such that, for each positive $T$, the intersection of $X$ with any ball of radius $M_{X}(T)$ centered at a point of $X$ contains a translate of every $T$-patch of $X$. Minimal sets are an important class because they can be viewed as a mathematical analogue of a "ground state" of a physical system. This notion of minimality coincides with minimality of an associated dynamical system with an $\mathbb{R}^{n}$-action, see Section 4. Radin [48]-[50], [53] defines a "ground state" for analogous models in statistical mechanics to be a set of configurations that is minimal and uniquely ergodic, i.e., strictly ergodic, ${ }^{2}$ under an $\mathbb{R}^{n}$-action for tiling systems or under a $\mathbb{Z}^{n}$-action

\footnotetext{
${ }^{2}$ For a Delone set $X$ of finite type, strict ergodicity implies that any $T$-patch occurs in $X$ with a positive limiting asymptotic density, so that $X$ has a well-defined diffraction measure (Fourier transform of autocorrelation measure), see [28]. Thus it has long-range order in the sense of part I. This diffraction measure need not have strong long-range order in the sense of having "Bragg peaks" (delta functions).
} 
for lattice systems. Our notion of what constitutes a "ground state" is thus slightly more inclusive than Radin's.

In Section 3 we give sufficient conditions for a set of local rules $\mathcal{L}$ under isometries to enforce structures which are Delone sets of finite type (Theorem 3.1). In Section 4 we show that these sufficient conditions can always be satisfied for minimal Delone sets of finite type, and so obtain:

Theorem 1.1. Let $X$ be a Delone set of finite type $X$ in $\mathbb{R}^{n}$ which is minimal. Then there exists a set of local rules $\mathcal{L}$ under isometries such that $X$ satisfies $\mathcal{L}$ and any other set $Y$ that satisfies $\mathcal{L}$ is a Delone set of finite type.

This result applies to a large variety of structures, because minimal sets are ubiquitous. Given any Delone set of finite type $X$ there always exists a minimal set $X^{\prime}$ which is a limit of larger and larger patches of $X$. That is, there is a sequence of translations $\left\{\mathbf{x}_{k}: k \geq 1\right\}$ such that, for each $T>0$,

$$
X^{\prime} \cap B(\mathbf{0} ; T)=\lim _{k \rightarrow \infty}\left(X-\mathbf{x}_{k}\right) \cap B(\mathbf{0} ; T),
$$

where the limit is taken pointwise. (This fact follows by a standard argument in topological dynamics, using Zorn's lemma, see p. 29 of [18].)

The configurational entropy of a Delone set of finite type $X$ in $\mathbb{R}^{n}$ is given by

$$
H_{c}(X):=\limsup _{T \rightarrow \infty} \frac{1}{T^{n}} \log \left(N_{X}^{*}(T)\right),
$$

where $N_{X}^{*}(T)$ counts the number of translation-inequivalent $T$-patches of $X$, see part I. The known constructions of Delone sets of finite type having perfect local rules apparently all have zero configurational entropy. This has been proved in special cases, ${ }^{3}$ notably for Penrose tilings, which contain at most $O\left(T^{2}\right)$ different $T$-patches, because all $T$-patches appear at least once inside any patch of radius $c T$, for $c=11.09$, see p. 563 of [25]. It is known that there exist Delone sets of finite type that are minimal but have positive configurational entropy, as follows in the one-dimensional case from a result of Furstenberg [17, Theorem III.2]. Such sets are covered by Theorem 1.1.

The "local rules" whose existence is shown by Theorem 1.1 do not necessarily identify a single local isomorphism class of structures. Let $\mathcal{L}_{T}(X)$ denote the set of all $T$ patches of $X$. It seems possible that the configurational entropy of the class of structures $\Sigma\left(\mathcal{L}_{T}(X)\right)$ produced by the local rules of radius $T$ can be larger than the configurational entropy $H_{c}(X)$ of the initial structure $X$. We conjecture that as the radius $T$ increases, the configurational entropy $H_{c}\left(\Sigma\left(\mathcal{L}_{T}(X)\right)\right)$ decreases to $H_{c}(X)$ as $T \rightarrow \infty$.

Theorem 1.1 shows that local rules under isometries can specify a wide variety of structures describable using a finite number $s$ of "internal dimensions," where $s$ is generally larger than the dimensionality $n$ of the space $\mathbb{R}^{n}$. It remains an open question whether every Delone set of finite type $X$ satisfies a set of local rules $\mathcal{L}$ which themselves can be satisfied only by Delone sets of finite type.

\footnotetext{
${ }^{3}$ See [16] and [19]. Related entropy zero results for lattice gas models appear in [49] and [51].
} 
In Section 4 we give several characterizations of minimal Delone sets. We define a concept of minimality of a Delone set $X$ under isometries, which we call isometryminimal, and show that it corresponds to minimality for a topological dynamical system with an $\mathcal{E}^{n}$-action, where $\mathcal{E}^{n}$ denotes the Euclidean group of isometries of $\mathbb{R}^{n}$. We prove that for Delone sets of finite type the properties of minimality and isometry-minimality coincide (Theorem 4.3). In the remainder of Section 4 we prove Theorem 1.1.

In Section 5 we study perfect local rules. A set $X$ has perfect local rules $\mathcal{L}$ under translations if all sets $Y$ that satisfy $\mathcal{L}$ under translations are locally isomorphic to $X$ under translations. Any set $X$ having perfect local rules is necessarily a Delone set of finite type that is minimal. We define perfect local rules $\mathcal{L}$ under isometries analogously: any two sets $X$ that satisfy $\mathcal{L}$ under isometries are locally isomorphic under isometries, i.e., they have the same set of $T$-neighborhoods under isometries, for each positive $T$. It is generally easier to verify the existence of perfect local rules under translations than to verify it for isometries. In Section 5 we prove:

Theorem 1.2. Let $X$ be a Delone set of finite type in $\mathbb{R}^{n}$. Then $X$ has perfect local rules under translations if and only if $X$ has perfect local rules under isometries.

Structures having perfect local rules under translations or under isometries seem to be extremely special. The known examples of sets having perfect local rules under translations make use of a self-similarity property, as described by Gähler [20]. Ideal crystals are included under such constructions, using a similarity factor which is an integer $m \geq 2$. There may well exist examples of structures having perfect local rules under translations that are based on self-affine constructions rather than self-similar constructions. The most general result on sets having perfect local rules under isometries is that of Goodman-Strauss [24], which proves that perfect local rules under isometries exist for nearly all substitution tilings.

There do exist Delone sets which have perfect local rules under isometries, but do not have perfect local rules under translations. One such example comes from the pinwheel tilings of Radin [52]. Another example comes from the vertices of the $S C D$-tiles in [12], for $S C D(m / n, s, h)$ with $n$ odd, using fact (13) in [12]. The quaquaversal tilings of Conway and Radin [9] can be suitably decorated to yield a Delone set with perfect local rules under isometries, using the result of Goodman-Strauss [24]. Theorem 1.2 implies that these structures are not ${ }^{4}$ Delone sets of finite type.

There have been many previous studies of "local rules" for quasicrystals. Much of this work has been based on tiling models for quasicrystals. In the Appendix we relate various notions of "local rules" and "matching rules" for tiling models to the concepts defined here for Delone set models. We include a formal definition of "perfect matching rules," which is a more general concept than "perfect local rules." The concept of "perfect matching rules" involves the notion of one tiling being locally derivable from another, which was introduced by Baake et al. [4]. Most previous work on tilings allows prototiles to be moved only by translations; in that case decorations of these tilings yield Delone sets of finite type. Several authors have developed "local rules" (under translations)

\footnotetext{
${ }^{4}$ This fact can be proved directly for these structures, without recourse to Theorem 1.2.
} 
for tilings which enforce stronger properties, for example, "quasiperiodicity," see, for example, [22], [29], and [36]-[38]. The use of Delone set models for certain quasicrystals and interconversions between Delone sets and tilings, particularly with "perfect matching rules," is discussed in [32].

Concerning physical quasicrystals it is now known that there are some materials (for example, $\mathrm{Al} \mathrm{Pd} \mathrm{Mn)} \mathrm{that} \mathrm{exhibit} \mathrm{a} \mathrm{quasicrystalline} \mathrm{phase} \mathrm{that} \mathrm{is} \mathrm{thermodynamically} \mathrm{sta-}$ ble, i.e., which at some temperatures and pressures is the structure that minimizes the free energy, see p. 236 of [23]. Recent proposals of Steinhardt and Jeong [60] present mechanisms to explain how Penrose tilings can arise as "energy-minimizing" arrangements of overlapping local configurations, and Burkov [7], [8] supplied similar results for tilings related to the Tubingen triangle tilings. These configurations are specified by a kind of "local rules under translations." The interpretation of "local rules" as representing energy-minimizing interactions at low temperature has been repeatedly proposed, see [42], Section 6 of [29], and [22].

A wide variety of other quasicrystalline materials are not known to be thermodynamically stable but instead are currently believed to be entropically stabilized. For entropically stabilized materials the possible configuations consist of a large number of nearly equal energy configurations which are interchangeable among themselves. These configurations minimize the free energy at the given temperature and pressure, in a competition between minimum energy and maximum entropy. We view these sets of configurations as being analogous to minimal sets $[[X]]_{\mathcal{E}}$ in Section 4 with positive configurational entropy. Sets of positive configurational entropy given by local rules may arise in "random tiling" models for quasicrystals, see [26] and [27].

The results of this paper demonstrate the existence of local rules under isometries that enforce weak long-range translational order. Our purpose here is only to show that such local rules exist, without giving reasonable bounds on the distances over which these local rules act. We were therefore able to make the simplifying assumption that all local rules consist of spherical neighborhoods. More flexible "local rules" that use nonspherical neighborhoods appear in the concept of a "Delone graph" introduced in [13], and in the "local rules" considered in [60].

Finally we observe that the results of this paper apply to more general solid state structures than those which have strong long-range order given by an X-ray diffraction pattern consisting entirely of delta functions ("Bragg peaks"). There are minimal Delone sets of finite type which do not have long-range order in this sense. It is therefore at least conceivable that there exist thermodynamically stable materials which have atomic positions described by a Delone set of finite type, which are not quasicrystalline in this sense. Such structures would not necessarily have X-ray diffraction patterns with any "Bragg peaks" from which the translational ordering can be inferred. Possibilities of this sort have been advocated by Danzer [11], see also Berend and Radin [5].

Notation. When $Y$ is a finite set, $|Y|$ denotes the number of elements of $Y$. If $Y$ is in $\mathbb{R}^{n}$, then $[Y]$ denotes the additive subgroup of $\mathbb{R}^{n}$ generated by the elements of $Y$; we regard $[Y]$ as a $\mathbb{Z}$-module.

The Euclidean group $\mathcal{E}^{n}$ consists of all (Euclidean) isometries of $\mathbb{R}^{n}$, i.e., of all motions $\varphi: \mathbb{R}^{n} \rightarrow \mathbb{R}^{n}$ given by

$$
\varphi(\mathbf{x})=Q \mathbf{x}+\mathbf{t}, \quad \text { with } \quad Q \in O(n, \mathbb{R}) \quad \text { and } \quad \mathbf{t} \in \mathbb{R}^{n},
$$


where $O(n, \mathbb{R})$ is the orthogonal group, i.e., $O(n, \mathbb{R})=\left\{M: M M^{t}=I\right\}$. The translation group $\mathcal{T}^{n}$ is the subgroup of $\mathcal{E}^{n}$ where $Q$ is the identity; we sometimes identify it with $\mathbb{R}^{n}$.

For an arbitrary set $Y$ in $\mathbb{R}^{n}$ we define its point symmetry group (at $\mathbf{x}=\mathbf{0}$ ) by

$$
\operatorname{Psym}(Y):=\{Q \in O(n, \mathbb{R}): Q Y=Y\}
$$

For a collection of sets $\mathcal{X}$ we analogously define $\operatorname{Psym}(\mathcal{X})=\{Q \in O(n, \mathbb{R}): Q \mathcal{X}=\mathcal{X}\}$.

The notations $[[X]]_{\mathcal{E}}$ and $[[X]]_{\mathcal{T}}$ denote the local isomorphism classes of $X$ under isometries and translations, respectively. For a collection of local rules $\mathcal{L}$, the sets $\Sigma_{\mathcal{E}}(\mathcal{L})$ and $\Sigma_{\mathcal{T}}(\mathcal{L})$ consist of all sets $Y$ that satisfy the local rules under isometries and translations, respectively. For a Delone set $X$, the sets $\Sigma_{\mathcal{E}}(X)$ and $\Sigma_{\mathcal{T}}(X)$ consist of all structures $Y$, each of whose $T$-neighborhoods is isometric (resp. translation-equivalent) to a $T$-neighborhood of $X$, for all $T>0$.

\section{Preliminary Results}

In this section we prove basic properties of Delone sets of finite type under translations and under isometries. We also relate the local isomorphism classes of $X$ under translations and isometries.

The concept of an enclosed point, which was introduced in [15], plays an important role in many proofs in this paper.

Definition 2.1. (i) A point $\mathbf{y} \in Y$ is called an enclosed point of $Y$ if it is in the interior of the convex hull of $Y$.

(ii) A set $Y$ is called an enclosing set if it contains an enclosed point.

For an enclosed point the vectors $\left\{\mathbf{y}^{\prime}-\mathbf{y}: \mathbf{y}^{\prime} \in Y\right\}$ span $\mathbb{R}^{n}$. It follows that if $Y$ is an enclosing set, then an isometry $\varphi$ of $\mathbb{R}^{n}$ is uniquely determined by the data $\mathbf{y} \rightarrow \varphi(\mathbf{y})$ for $\mathbf{y} \in Y$.

Definition 2.2. (i) A point $\mathbf{x} \in X$ is R-enclosed if it is an enclosed point of the set $X \cap B(\mathbf{x} ; R)$.

(ii) A point $\mathbf{x}$ in a set $X$ is an $R$-point or is strongly $R$-enclosed if each closed ball of radius $R$ that contains $\mathbf{x}$ on its boundary also contains another point of $X$.

\section{Lemma 2.1.}

(i) Any strongly $R$-enclosed point $\mathbf{x} \in X$ is R-enclosed.

(ii) If every point $\mathbf{x} \in X$ is strongly $R$-enclosed, then $X$ is relatively dense with constant $R$, and conversely.

Proof. See Lemma 3.2 of [15].

We define "local" properties of sets $X$ under isometries and under translations. We first consider isometries. 
Definition 2.3. (i) A $T$-star is any $T$-neighborhood $\mathcal{P}_{X}(\mathbf{x} ; T)$ of some $\mathbf{x} \in X$, viewed as being movable by isometries. We call $\mathbf{x}$ the center of the $T$-star. A centered T-star $\mathcal{P}$ is obtained by translating it to the origin, i.e.,

$$
\mathcal{P}:=X \cap B(\mathbf{x} ; T)-\mathbf{x}
$$

(ii) The T-atlas of $X$ under isometries, denoted $\mathcal{A}_{X}^{\mathcal{E}}(T)$, is the set of all isometry equivalence classes of $T$-stars of $X$.

Definition 2.4. Two sets $X$ and $X^{\prime}$ are locally isomorphic under isometries if $X$ contains an isometric copy of each $T$-star of $X^{\prime}$ and vice-versa, i.e., if

$$
\mathcal{A}_{X}^{\mathcal{E}}(T)=\mathcal{A}_{X^{\prime}}^{\mathcal{E}}(T), \quad \text { for each } \quad T>0 .
$$

We let $[[X]]_{\mathcal{E}}$ denote the collection of all sets $X^{\prime}$ locally isomorphic to $X$ under isometries. There is a partial order $\leq_{\mathcal{E}}$ on such local isomorphism classes, in which

$$
[[X]]_{\mathcal{E}} \leq_{\mathcal{E}}\left[\left[X^{\prime}\right]\right]_{\mathcal{E}}
$$

provided that, for each $T>0, X^{\prime}$ contains an isometric copy of every $T$-star of $X$.

Definition 2.5. A local isomorphism class $[[X]]_{\mathcal{E}}$ is isometry-minimal if it is minimal in the partial order $\leq_{\mathcal{E}}$. We also say that a set $X$ is isometry-minimal if $[[X]]_{\mathcal{E}}$ is isometryminimal.

Definition 2.6. A Delone set $X$ is of isometry-finite type or is of finite type under isometries if $\left|\mathcal{A}_{X}^{\mathcal{E}}(T)\right|$ is finite for all $T>0$.

These sets are characterized by the following finiteness condition.

Theorem 2.1. Let $X$ be a Delone set in $\mathbb{R}^{n}$ with relative denseness constant $R$. Then $X$ is a Delone set of isometry-finite type if and only if it has finitely many isometry classes of $3 R$-neighborhoods, i.e., if and only if $\left|\mathcal{A}_{X}^{\mathcal{E}}(3 R)\right|$ is finite.

Proof. One direction is clear. For the other direction, suppose that $\left|\mathcal{A}_{X}^{\mathcal{E}}(3 R)\right|$ is finite, and we must show for each $T>0$ that $X$ has only finitely many $T$-neighborhoods up to isometries. We may suppose $\mathbf{0} \in X$ by translating $X$ if necessary. By hypothesis there are a finite number of choices for the $3 R$-neighborhood $\mathcal{P}_{X}(\mathbf{0} ; 3 R)$, up to isometry. Let this neighborhood be chosen. We show there are only finitely many possible ways to extend it to a neighborhood $\mathcal{P}_{X}(\mathbf{0} ; T)$. For each point $\mathbf{x} \in \mathcal{P}_{X}(\mathbf{0} ; 3 R)$ with $\|\mathbf{x}\|<2 R$, its $R$-neighborhood

$$
Y:=X \cap B(\mathbf{x} ; R) \subseteq \mathcal{P}_{X}(\mathbf{0} ; 3 R)
$$

is completely determined by $\mathcal{P}_{X}(\mathbf{0} ; 3 R)$. By Lemma $2.2 Y$ is an enclosing set of $\mathbf{x}$, so $\left\{\mathbf{x}^{\prime}-\mathbf{x}: \mathbf{x}^{\prime} \in Y\right\}$ spans $\mathbb{R}^{n}$. For a given $Q \in O(n, \mathbb{R})$, the images $\left\{Q \mathbf{x}^{\prime}: \mathbf{x}^{\prime} \in Y\right\}$ determine $Q$ uniquely, hence it follows that there are only finitely many ways to extend 
$Y$ to a $3 R$-neighborhood of $\mathbf{x}$ using a set isometric to one of the sets in $\mathcal{A}_{X}^{\mathcal{E}}(3 R)$. By the Delone property the resulting $3 R$-neighborhoods for all such $\mathbf{x}$ cover a larger region which includes $B(\mathbf{0} ; 4 R)$. We now proceed by induction on $k$, for $k \geq 4$, to extend to $X \cap B(\mathbf{0} ;(k+1) R)$ given $X \cap B(\mathbf{0} ; k R)$, making a finite number of choices of cardinality bounded in terms of $n, r, R$, and $k$, using the Delone property of $X$. Continuing in this way we eventually determine $\mathcal{P}_{X}(\mathbf{0} ; T)$, while making finitely many choices in all, so $\left|\mathcal{A}_{X}^{\mathcal{E}}(T)\right|$ is finite. Thus $X$ is a Delone set of isometry-finite type.

We now define analogous concepts for equivalence under translations.

Definition 2.7. (i) A $T$-patch $\mathcal{P}$ is any $T$-neighborhood of $\mathbf{x} \in X$, regarded as movable by translations. A centered $T$-patch is a $T$-patch translated to the origin, again given by (2.1).

(ii) The T-atlas of $X$ (under translations), denoted $\mathcal{A}_{X}(T)$, is the set of translation equivalence classes of $T$-patches of $X$.

Definition 2.8. Two sets $X$ and $X^{\prime}$ are locally isomorphic (under translations) if $X$ contains a translate of each $T$-neighborhood of $X^{\prime}$ and vice-versa, i.e., if

$$
\mathcal{A}_{X}(T)=\mathcal{A}_{X^{\prime}}(T), \quad \text { for each } \quad T>0 .
$$

We let $[[X]]_{\mathcal{T}}$ denote the collection of all sets $X^{\prime}$ locally isomorphic to $X$ under translations. We have a partial order $\leq_{\mathcal{T}}$ on such sets, where

$$
[[X]]_{\mathcal{T}} \leq \mathcal{T}\left[\left[X^{\prime}\right]\right]_{\mathcal{T}}
$$

means that the set $X^{\prime}$ contains a translate of every $T$-patch of $X$, for each $T>0$.

Definition 2.9. A local isomorphism class $[[X]]_{\mathcal{T}}$ is minimal (under translations) if it is minimal in the partial order $\leq_{\mathcal{T}}$. We also say that $X$ is minimal (under translations) if $[[X]]_{\mathcal{T}}$ is minimal.

In part I it was shown that a set $X$ is a Delone set of finite type if and only if $\left|\mathcal{A}_{X}(T)\right|$ is finite for all $T>0$.

Theorem 2.2. Let $X$ be a Delone set in $\mathbb{R}^{n}$ with relative denseness constant $R$. Then $X$ is a Delone set of finite type (under translations) if and only if it has finitely many translation-equivalence classes of $2 R$-neighborhoods, i.e., if and only if $\left|\mathcal{A}_{X}(2 R)\right|$ is finite.

Proof. This result for $3 R$-neighborhoods can be proved similarly to Theorem 2.1. A proof for $2 R$-neighborhoods appears in Theorem 2.1 of part I.

We clarify the relation between translation and isometry local isomorphism classes $[[X]]_{\mathcal{T}}$ and $[[X]]_{\mathcal{E}}$ in the next two lemmas. Recall that for a collection of sets $\mathcal{X}$ the group $\operatorname{Psym}(\mathcal{X})$ is the set of rotations $Q \in O(n, \mathbb{R})$ for which $Q \mathcal{X}=\mathcal{X}$. 
Lemma 2.2. Suppose that $X$ is a Delone set of finite type in $\mathbb{R}^{n}$. Then the group $\operatorname{Psym}\left([[X]]_{\mathcal{T}}\right)$ of rotations that leave $[[X]]_{\mathcal{T}}$ invariant is finite.

Proof. Pick $Y \in[[X]]_{\mathcal{T}}$ with $\mathbf{0} \in Y$. If $Q \in \operatorname{Psym}\left([[X]]_{\mathcal{T}}\right)$, then $Q Y \in[[X]]_{\mathcal{T}}$ as $Q\left(\mathcal{P}_{X}(\mathbf{0} ; 2 R)\right)$ must be in the $2 R$-atlas $\mathcal{A}_{X}(2 R)$. Now $\mathcal{P}_{X}(\mathbf{0} ; 2 R)$ is an enclosing set of $\mathbf{0}$, so the isometry $Q$ is uniquely determined by the images of the points in $\mathcal{P}_{X}(\mathbf{0} ; 2 R)$. Since $\left|\mathcal{A}_{X}(2 R)\right|$ is finite, and each patch in $\mathcal{A}_{X}(2 R)$ contains finitely many points, there are only finitely many possibilities for $Q$.

An ideal crystal $X$ in $\mathbb{R}^{n}$ is any set that consists of a finite number of translates of a full rank lattice in $\mathbb{R}^{n}$. In this case Psym $\left([[X]]_{\mathcal{T}}\right)$ is the point group of the ideal crystal $X$, and it is well known that there is an upper bound on the order $\left|P \operatorname{sym}\left([[X]]_{\mathcal{T}}\right)\right|$ that depends only on the dimension $n$. This fact does not hold for general Delone sets of finite type; in each dimension $n \geq 2$ one can construct examples $X$ in which $\left|\operatorname{Psym}\left([[X]]_{\mathcal{T}}\right)\right|$ is arbitrarily large.

For any Delone set $X$ in $\mathbb{R}^{n}$, we have the inclusion

$$
O(n, \mathbb{R})[[X]]_{\mathcal{T}} \subseteq[[X]]_{\mathcal{E}},
$$

in which $O(n, \mathbb{R})$ is the orthogonal group in $\mathbb{R}^{n}$. We say that $X$ is rigid if equality holds in (2.6).

Lemma 2.3. Any Delone set $X$ in $\mathbb{R}^{n}$ of finite type is rigid. That is,

$$
[[X]]_{\mathcal{E}}=O(n, \mathbb{R})[[X]]_{\mathcal{T}} .
$$

Proof. Let $Y \in[[X]]_{\mathcal{E}}$, and without loss of generality we may translate $Y$ so that $\mathbf{0} \in Y$. We wish to show that there exists $Q \in O(n, \mathbb{R})$ and $\tilde{X} \in[[X]]_{\mathcal{T}}$ such that

$$
Y=Q \tilde{X}
$$

Since each $T$-neighborhood of $Y$ around $\mathbf{0}$ is isometric to some $T$-neighborhood of $X$, for each integer $k>R$ we can find $Q_{k} \in O(n, \mathbb{R})$ and $\mathbf{x}_{k} \in X$ such that

$$
Y \cap B(\mathbf{0} ; k)=Q_{k}\left(X-\mathbf{x}_{k}\right) \cap B(\mathbf{0} ; k),
$$

and, in particular,

$$
Y \cap B(\mathbf{0} ; R)=Q_{k}\left(X-\mathbf{x}_{k}\right) \cap B(\mathbf{0}, R) .
$$

Fix $k_{0}=\lfloor R\rfloor+1$. For any $k \geq k_{0}$, the last equality gives

$$
\left(X-\mathbf{x}_{k}\right) \cap B(\mathbf{0} ; R)=Q_{k}^{-1} Q_{k_{0}}\left(X-\mathbf{x}_{k_{0}}\right) \cap B(\mathbf{0} ; R) .
$$

We claim that there are only finitely many choices for

$$
Q:=Q_{k}^{-1} Q_{k_{0}} \in O(n, \mathbb{R})
$$


as $k$ varies over all $k \geq k_{0}$. To see this, note first that the finite set $X_{0}:=\left(X-\mathbf{x}_{k_{0}}\right) \cap B(\mathbf{0} ; R)$ is an enclosing set because $X$ is $R$-relatively dense. It follows that the points $\left\{\mathbf{x} \in X_{0}\right\}$ span $\mathbb{R}^{n}$, hence the image $\mathbf{x} \rightarrow Q \mathbf{x}$ for $\mathbf{x} \in X_{0}$ determines $Q$ uniquely. Second, the set $(X-X) \cap B(\mathbf{0} ; R)$ is a finite set because $X$ is a Delone set of finite type. Now for each $\mathbf{x}-\mathbf{x}_{k_{0}} \in X_{0}$ there exists $\mathbf{x}^{\prime} \in X$ such that

$$
\mathbf{y}^{\prime}:=\mathbf{x}^{\prime}-\mathbf{x}_{k}=Q\left(\mathbf{x}-\mathbf{x}_{k_{0}}\right)
$$

hence

$$
\mathbf{y}^{\prime} \in\left(X-\mathbf{x}_{k}\right) \cap B(\mathbf{0} ; R) \subseteq(X-X) \cap B(\mathbf{0} ; R) .
$$

There are only finitely many maps $\varphi: X_{0} \rightarrow(X-X) \cap B(0 ; R)$, hence there are only finitely many choices for $Q$.

We can now pick an infinite subsequence $\left\{k_{j}: 1 \leq j \leq \infty\right\}$ with $k_{j} \rightarrow \infty$ on which $Q_{k_{j}}=Q$ is constant. We then have

$$
Y \cap B\left(\mathbf{0} ; k_{j}\right)=Q\left(X-\mathbf{x}_{k_{j}}\right) \cap B\left(\mathbf{0} ; k_{j}\right), \quad \text { all } \quad j \geq 1 .
$$

The pointwise limit

$$
\tilde{X}:=\lim _{j \rightarrow \infty}\left(X-\mathbf{x}_{k_{j}}\right) \cap B\left(\mathbf{0} ; k_{j}\right)
$$

is a well-defined set by (2.11). Clearly, $\tilde{X} \in[[X]]_{\mathcal{T}}$, and (2.11) implies that $Y=Q \tilde{X}$.

\section{Local Rules}

We use the term "local rules" to describe a set of local restrictions on the points in a Delone set $X$.

Definition 3.1. (i) A set of local rules $\mathcal{L}$ of radius $\rho$ is a finite collection of discrete sets $\left\{Y_{i}\right\}$ containing $\mathbf{0}$ which are contained in the open ball $B(\mathbf{0} ; \rho)$.

(ii) A set $X$ satisfies the local rules $\mathcal{L}$ under isometries if each $\rho$-neighborhood $\mathcal{P}_{X}(\mathbf{x} ; \rho)$ of $X$ is isometric to a set in $\mathcal{L}$ under an isometry that sends $\mathbf{x}$ to $\mathbf{0}$. We set

$$
\Sigma_{\mathcal{E}}(\mathcal{L}):=\{Y: Y \text { satisfies } \mathcal{L} \text { under isometries }\} .
$$

(iii) A set $X$ satisfies the local rules $\mathcal{L}$ (under translations) if each $\rho$-neighborhood $\mathcal{P}_{X}(\mathbf{x} ; \rho)$ of $X$ when translated to $\mathbf{0}$ is a set in $\mathcal{L}$. We set

$$
\Sigma_{\mathcal{T}}(\mathcal{L}):=\{Y: Y \text { satisfies } \mathcal{L} \text { under translations }\}
$$

Note that if $Y \in \Sigma_{\mathcal{E}}(\mathcal{L})$, then its local isomorphism class $[[Y]]_{\mathcal{E}} \subseteq \Sigma_{\mathcal{E}}(\mathcal{L})$. Furthermore, $\Sigma_{\mathcal{E}}(\mathcal{L})$ is closed downward under the ordering $\leq \mathcal{E}$, namely, if $[[Y]]_{\mathcal{E}} \subseteq \Sigma_{\mathcal{E}}(\mathcal{L})$ and $\left[\left[Y^{\prime}\right]\right]_{\mathcal{E}} \leq \leq_{\mathcal{E}}[[Y]]_{\mathcal{E}}$, then $\left[\left[Y^{\prime}\right]\right]_{\mathcal{E}} \subseteq \Sigma_{\mathcal{E}}(\mathcal{L})$. It follows that if $\Sigma_{\mathcal{E}}(\mathcal{L})=[[X]]_{\mathcal{E}}$, then $X$ is isometry-minimal.

We similarly have that if $Y \in \Sigma_{\mathcal{T}}(\mathcal{L})$, then $[[Y]]_{\mathcal{T}} \subseteq \Sigma_{\mathcal{T}}(\mathcal{L})$, and $\Sigma_{\mathcal{T}}(\mathcal{L})$ is closed downward under the ordering $\leq_{\mathcal{T}}$. It follows that if $\Sigma_{\mathcal{T}}(\mathcal{L})=[[X]]_{\mathcal{T}}$, then $X$ is minimal. 
The property of being a Delone set is enforceable by local rules under isometries. We say that a set of local rules $\mathcal{L}$ of radius $\rho \geq R$ is strongly $R$-enclosing if $\mathbf{0}$ is a strongly enclosed $R$-point of each $Y_{i} \in \mathcal{L}$.

Lemma 3.1. Let $\mathcal{L}$ be a set of local rules, of radius $\rho \geq R$, such that $\mathcal{L}$ is strongly $R$-enclosing. Then any set $X$ that satisfies $\mathcal{L}$ under isometries is a Delone set whose relative denseness constant is at most $R$.

Proof. First, $X$ is uniformly discrete, because there is a lower bound $r$ of closeness between any two points in any $Y_{i}$ in $\mathcal{L}$, because $\mathcal{L}$ is finite. Second, to show that $X$ is relatively dense with constant $R$, we argue by contradiction. Suppose not, and take some empty ball $B(\mathbf{z} ; T)$ with $T \geq R$. Choose a point $\mathbf{x} \in X$ that is a closest point to $\mathbf{z}$ and translate the ball in the direction $\mathbf{x}-\mathbf{z}$ until $\mathbf{x}$ is on its boundary. By the triangle inequality, the resulting ball $B\left(\mathbf{z}^{\prime} ; T\right)$ contains no point of $X \backslash\{\mathbf{x}\}$, for any such point would be closer to $\mathbf{z}$ than to $\mathbf{x}$. This contradicts $\mathbf{x}$ being an $R$-point of $X$, which it must be since $\mathcal{L}$ is strongly $R$-enclosing.

This result immediately extends to give local rules that enforce the property of being a Delone set of isometry-finite type.

Lemma 3.2. Let $\mathcal{L}$ be a set of local rules of radius $\rho \geq 3 R$, such that each set $Y_{i}$ in $\mathcal{L}$ is strongly $R$-enclosing. Then any set $X$ that satisfies $\mathcal{L}$ under isometries is a Delone set of isometry-finite type. Conversely, if $X$ is a Delone set of isometry-finite type, then its set $\mathcal{L}$ of centered $3 R$-stars is a set of local rules in which each set $Y_{i}$ in $\mathcal{L}$ is strongly $R$-enclosing.

Proof. By Lemma $3.1 X$ is a Delone set with relative denseness constant $R$. Since $\rho \geq 3 R$ and all its $3 R$-stars are isometric to sets in $\mathcal{L}$, it follows from Theorem 2.1 that $X$ is of isometry-finite type.

There is an analogous result for local rules under translations.

Lemma 3.3. Let $\mathcal{L}$ be a set of local rules of radius $\rho \geq 2 R$, such that each $Y_{i} \in \mathcal{L}$ is strongly $R$-enclosing. Then any set $X$ that satisfies $\mathcal{L}$ under translations is a Delone set of finite type. Conversely, if $\mathcal{L}$ is a Delone set of finite type, then its set $\mathcal{L}$ of centered $2 R$-patches is a set of local rules in which each $Y_{i}$ in $\mathcal{L}$ is strongly $R$-enclosing.

Proof. Similar to Lemma 3.2, using Theorem 2.2 instead of Theorem 2.1.

Lemma 3.2 shows that for each Delone set of finite type $X$ there exists a set of local rules $\mathcal{L}$ with the property that the set $\Sigma_{\mathcal{E}}(\mathcal{L})$ contains only Delone sets of isometry-finite type. To prove that for a specific $\mathcal{L}$ all elements of $\Sigma_{\mathcal{E}}(\mathcal{L})$ are Delone sets of finite type requires proving a finiteness result for the set of all rotations used in applying the elements of $\mathcal{L}$. This appears to be a nontrivial problem, because for some Delone sets of finite type $X$ in $\mathbb{R}^{n}$ the set of rotations $P \operatorname{sym}([X-X])$ that leave the $\mathbb{Z}$-module $[X-X]$ 
invariant may contain elements of infinite order if $n \geq 2$, and may be infinitely generated if $n \geq 3$, see [47] and [39].

We give below sufficient conditions on a set of local rules $\mathcal{L}$ for every set in $\Sigma_{\mathcal{E}}(\mathcal{L})$ to be a Delone set of finite type. These conditions force every set $X \in \Sigma_{\mathcal{E}}(\mathcal{L})$ to contain a relatively dense set of copies of a particular finite configuration $\Delta$ under isometries, such that these copies occur only in a finite number of orientations.

Definition 3.2. Let $\Delta$ be a finite set in $\mathbb{R}^{n}$ and let $G$ be a finite subgroup of $O(n, \mathbb{R})$. We say that $\Delta$ is a watermark with respect to $G$ for a set of local rules $\mathcal{L}$ under isometries if:

(i) $\mathbf{0} \in \Delta$ and $\mathbf{0}$ is a $T$-enclosed point of $\Delta$ for some $T \leq \frac{1}{2} \rho$, where $\rho$ is the radius of the local rules $\mathcal{L}$. Call $\mathbf{0}$ the center of $\Delta$.

(ii) Each $Y_{i} \in \mathcal{L}$ contains an isometric copy of $\Delta$ in $Y_{i} \cap B(\mathbf{0} ; \rho-T)$.

(iii) A set $Y_{i} \in \mathcal{L}$ is $\Delta$-centered if it contains an isometric copy of $\Delta$ centered at $\mathbf{0}$, i.e., it contains $\Delta^{\prime}=Q \Delta$ for some $Q \in O(n, \mathbb{R})$. For each $\Delta$-centered $Y_{i}$, every isometric copy $\Delta^{\prime \prime}$ of $\Delta^{\prime}$ contained in $Y_{i}$ satisfies

$$
\Delta^{\prime \prime}=Q^{\prime \prime} \Delta^{\prime}+\mathbf{t}^{\prime \prime}, \quad \text { with } \quad Q^{\prime \prime} \in G \quad \text { and } \quad \mathbf{t}^{\prime \prime} \in \mathbb{R}^{n} .
$$

(iv) For each $\Delta$-centered $Y_{i}$ in $\mathcal{L}$, the centers $\left\{\mathbf{t}^{\prime \prime}\right\}$ of all isometric copies of $\Delta$ contain $\mathbf{0}$ as a strongly $\frac{1}{2} \rho$-enclosed point.

A $\Delta$-centered $Y_{i}$ may contain several distinct copies $\Delta^{\prime}$ of $\Delta$ centered at $\mathbf{0}$, and these copies must differ by elements of $G$. In particular, the group $G$ must contain the group $\operatorname{Psym}(\Delta)$. (The $T$-enclosing property of $\Delta$ guarantees that $P \operatorname{sym}(\Delta)$ is a finite group.) In principle, one can check by a finite computation ${ }^{5}$ whether a finite set $\mathcal{L}$ has some $(\Delta, G)$ such that $\Delta$ is a watermark with respect to $G$ for $\mathcal{L}$.

Definition 3.3. Let a finite set $\mathcal{L}$ have a pair $(\Delta, G)$ as a watermark. We define the normalization $\mathcal{L}_{\Delta}$ of $\mathcal{L}$ with respect to $(\Delta, G)$ to be the set of local rules obtained from $\mathcal{L}$ by replacing each $\Delta$-centered $Y_{i}$ by the set of all $Q Y_{i}$ for $Q$ in $O(n, \mathbb{R})$ such that $Q Y_{i}$ contains $\Delta$ centered at $\mathbf{0}$.

It is clear that the normalization $\mathcal{L}_{\Delta}$ is a finite set and that the local rules $\mathcal{L}$ and $\mathcal{L}_{\Delta}$ under isometries give the same sets.

Theorem 3.1. If $\mathcal{L}$ is a set of local rules of radius $\rho$ that contains a watermark $\Delta$ with respect to $G$, then

$$
\Sigma_{\mathcal{E}}(\mathcal{L})=O(n, \mathbb{R}) \Sigma_{\mathcal{T}}\left(G \mathcal{L}_{\Delta}\right)
$$

so that $\Sigma_{\mathcal{E}}(\mathcal{L})$ consists entirely of Delone sets of finite type. Furthermore, each

\footnotetext{
${ }^{5}$ Here we assume given a "black box" algorithm that can decide whether two given interpoint vectors in $\mathbb{R}^{n}$ are equal.
} 
$X \in \Sigma_{\mathcal{E}}(\mathcal{L})$ contains a relatively dense set of isometric copies of $\Delta$ and has

$$
\operatorname{Psym}\left([[X]]_{\mathcal{T}}\right) \subseteq G .
$$

Proof. The inclusion

$$
O(n, \mathbb{R}) \Sigma_{\mathcal{T}}\left(G \mathcal{L}_{\Delta}\right) \subseteq \Sigma_{\mathcal{E}}(\mathcal{L})
$$

is clear. For the other direction, suppose that $X \in \Sigma_{\mathcal{E}}(\mathcal{L})$. Since by property (ii) of Definition 3.2 each rule $Y_{i} \in \mathcal{L}$ contains an isometric copy $\Delta^{\prime}$ of $\Delta$, so does $X$. For convenience, we first shift $X$ by a global isometry to $\tilde{X}=Q X+\mathbf{t}$, in such a way that $\Delta^{\prime}$ is moved to $\Delta$, and view $\Delta$ as "marked" in $\tilde{X}$. It suffices to prove that $\tilde{X}$ is a Delone set of finite type.

Let $\mathcal{L}_{\Delta}$ be the normalization of $\mathcal{L}$ and set

$$
\tilde{\mathcal{L}}_{\Delta}:=G \mathcal{L}_{\Delta}=\left\{g Y: Y \in \mathcal{L}_{\Delta}, g \in G\right\} .
$$

We show that $\tilde{X}$ can be built up using local rules from $\tilde{\mathcal{L}}_{\Delta}$ under translations, starting from the "marked" set $\Delta$ centered at $\mathbf{0}$. We proceed by induction on the number of "marked" isometric copies of $\Delta$. The $\rho$-neighborhood of $\mathbf{0}$ is isometric to some set in $\mathcal{L}$, necessarily $\Delta$-centered, hence it must be equal to some set in $\tilde{\mathcal{L}}_{\Delta}$, according to property (iii) of Definition 3.2. Now (3.3) implies that every isometric copy of $\Delta$ in $B(\mathbf{0} ; \rho)$ is translation-equivalent to

$$
Q \Delta \quad \text { for some } Q \in G .
$$

We "mark" all these copies of $\Delta$ having centers within distance $\frac{1}{2} \rho$ of $\mathbf{0}$, and let their center locations form a list $\mathcal{C}_{1}$ of eligible $\Delta$-centers, and let the "already inspected" list $\mathcal{M}_{1}=\{\mathbf{0}\}$. The induction hypothesis will be that $\mathcal{C}_{k}$ is nonempty and only includes centers of copies of $\Delta$ that satisfy (3.8). At the $(k+1)$ st stage we take the first element of the list $\mathbf{x}_{k}$, and find an element of $\mathcal{L}$ that agrees with $\tilde{X}$ on its $\rho$-neighborhood. This element $\mathbf{x}_{k}$ is in $\tilde{\mathcal{L}}_{\Delta}$ and by the induction hypothesis it is translation-equivalent to an element of $\tilde{\mathcal{L}}_{\Delta}$, since $G$ is a group. Let $\mathcal{C}_{k+1}$ be obtained from $\mathcal{C}_{k}$ by removing $\mathbf{x}_{k}$ and adding any new $\Delta$-centers within distance $\frac{1}{2} \rho$ of $\mathbf{x}$ to the end of this list. We update the "already inspected" list to $\mathcal{M}_{k+1}=\mathcal{M}_{k} \cup\left\{\mathbf{x}_{k}\right\}$. Any new $\Delta$-centers added to $\mathcal{C}_{k+1}$ satisfy (3.8) by condition (iii) of Definition 3.2. The ordered list $\mathcal{C}_{k+1}$ cannot be empty, for if it were, then $\mathcal{M}_{k+1}$ would have the property that every element in it was a $\frac{1}{2} \rho$-point, by property (iv) of Definition 3.2. Then Lemma 2.1 implies that $\mathcal{M}_{k+1}$ is $\frac{1}{2} \rho$-relatively dense, hence infinite, which contradicts $\left|\mathcal{M}_{k+1}\right|=k+1$. Now consider the complete "eventually inspected" set

$$
\tilde{\mathcal{M}}:=\bigcup_{k=1}^{\infty} \mathcal{M}_{k}=\bigcup_{k=1}^{\infty} \mathcal{C}_{k}
$$

Every element of $\tilde{\mathcal{M}}$ is a $\Delta$-center of $\tilde{X}$, and by property (iv) of Definition $3.2 \mathbf{x}_{k} \in \mathcal{M}_{k}$ is a $\frac{1}{2} \rho$-enclosed point of $\mathcal{M}_{k} \cup \mathcal{C}_{k+1}$ Thus every point of $\mathcal{M}$ is $\frac{1}{2} \rho$-enclosed, hence by Lemma $2.1 \tilde{\mathcal{M}}$ is $\frac{1}{2} \rho$-relatively dense.. However, each element of $\tilde{X}$ is specified by an 
element of $\tilde{\mathcal{L}}_{\Delta}$ centered at $X$, of radius $\rho$. Since all elements of $\tilde{\mathcal{M}}$ are $\Delta$-centered and satisfy (3.8), the same holds for every isometric copy $\tilde{\Delta}$ of $\Delta$ centered at $\mathbf{x}^{\prime} \in \tilde{X}$, by virtue of property (iii) of Definition 3.2 applied at some $\mathbf{x} \in \tilde{\mathcal{M}}$, with $\left\|\mathbf{x}^{\prime}-\mathbf{x}\right\| \leq \frac{1}{2} \delta$. The $\rho$-stars at all $\mathbf{x} \in \tilde{\mathcal{M}}$ cover $\tilde{X}$ and are each a translate of an element of $\tilde{\mathcal{L}}_{\Delta}$, hence $\tilde{X}$ contains only finitely many classes of $\rho$-stars under translation. Since $\tilde{X}$ is Delone with relative denseness constant $R \leq \frac{1}{2} \rho$, it is a Delone set of finite type by Theorem 2.2.

Finally, $\Delta$ is an enclosing set, so the image of its points under an isometry determines the isometry uniquely. Since all isometric copies of $\Delta$ in any set $X$ satisfy (3.8), we must have

$$
\operatorname{Psym}\left([[X]]_{\mathcal{T}}\right) \subseteq G
$$

as required.

Remarks. (1) In Section 4 we show that any Delone set of finite type that is minimal satisfies a set of local rules $\mathcal{L}$ having a watermark $(\Delta, G)$ to which Theorem 3.1 applies.

(2) We defined "watermark" above so that every isometric copy of $\Delta$ in $X$ is "marked." One can prove a strengthening of Theorem 3.1 in which only some copies of $\Delta$ are "marked," by changing the definition of "watermark" (Definition 3.2(iii)) to have (3.3) apply only to those copies $\Delta^{\prime \prime}$ for which $|t|=r_{i}$ for some fixed set of radius values

$$
0=r_{0}<r_{1}<\cdots<r_{m}<\rho-T
$$

and by changing Definition 3.2(iv) that $\mathbf{0}$ be a $\frac{1}{2} \rho$-enclosed point of the set of all $\left\{\mathbf{t}^{\prime \prime}\right\}$ with $\left|\mathbf{t}^{\prime \prime}\right|=r_{i}$ for some $i, 0 \leq i \leq m$.

\section{Minimality}

The notion of "minimality" arose in topological dynamics. The introduction of ergodic theory in lattice gas models in statistical mechanics goes back to work of Ruelle and Sinai around 1970. Later Radin [48], [49], [51], [53] pioneered the use of dynamical systems with a $\mathbb{Z}^{n}$-action, $\mathbb{R}^{n}$-action, or an $\mathcal{E}^{n}$-action in studying the structure of "ground states," for tilings and quasicrystalline structures. Dynamical systems with an $\mathbb{R}^{n}$-action were used in studying tiling models for quasicrystalline structures in [55] and [59], and dynamical systems with an $\mathcal{E}^{n}$-action were used in [54].

Recall that a Delone set of isometry-finite type $X$ is defined to be isometry-minimal if $[[X]]_{\mathcal{E}}$ is minimal in the partial ordering $\leq_{\mathcal{E}}$, and that a Delone set of finite type $X$ is defined to be minimal if $[[X]]_{\mathcal{T}}$ is minimal in the partial ordering $\leq_{\mathcal{T}}$. The first part of this section relates these two types of minimality to topological dynamical systems with an $\mathcal{E}^{n}$-action and an $\mathbb{R}^{n}$-action, respectively (Theorems 4.1 and 4.2). These equivalences are standard in the dynamical systems literature, and are included here to make the paper self-contained. The second part of this section shows for Delone sets of finite type that these two concepts of minimality coincide (Theorem 4.3), and then applies this result to prove Theorem 1.1.

We first give several equivalent conditions for isometry-minimality. 
Definition 4.1. (i) A Delone set $X$ is isometry-repetitive if for each positive $T$ there is a radius $R^{\prime}$ depending on $T$ such that each $R^{\prime}$-neighborhood of $X$ contains an isometric copy of each $T$-star of $X$.

(ii) The isometry-repetivity function $M_{X}^{\mathcal{E}}(T)$ of $X$ is the function that gives the least such value of $R^{\prime}$ for each $T>0$.

This definition is equivalent to $X$ being a Delone set of isometry-finite type, such that the set of isometric copies of any $T$-star occurring in $X$ is relatively dense.

We construct a topological dynamical system with an $\mathcal{E}^{n}$-action associated to an arbitrary Delone set $X$. Let $\mathcal{D}_{r, R}$ be the collection of all Delone sets with parameters $(r, R)$. We topologize $\mathcal{D}_{r, R}$ using the natural topology, which is defined by a metric

$$
\operatorname{dist}(X, \tilde{X}):=\sum_{\mathbf{x} \in X} 2^{-\|\mathbf{x}\|} \operatorname{dist}(\mathbf{x}, \tilde{X})+\sum_{\tilde{\mathbf{x}} \in \tilde{X}} 2^{-\|\tilde{\mathbf{x}}\|} \operatorname{dist}(\tilde{\mathbf{x}}, X) .
$$

That is, two Delone sets $X$ and $\tilde{X}$ are close in this metric if in some large ball of radius $T$ around the origin the points of $X$ and $\tilde{X}$ are in one-to-one correspondence, with each pair of points separated by distance at most $\varepsilon$. It is easy to check that this is a metric, and that $\mathcal{D}_{r, R}$ is a complete metric space and is compact. (That is, every sequence $\left\{\mathbf{x}_{k}: k \geq 1\right\}$ has a limit point in $\mathcal{D}_{r, R}$.) Note also that $\mathcal{D}_{r, R}$ is invariant under the action of the Euclidean group $\mathcal{E}^{n}$ of all isometries.

Definition 4.2. (i) Given a Delone set $X$, let $\mathcal{O}_{\mathcal{E}}(X)$ denote the orbit of $X$ under isometries $\mathcal{E}^{n}$, i.e.,

$$
\mathcal{O}_{\mathcal{E}}(X):=\left\{\varphi(X): \varphi \in \mathcal{E}^{n}\right\}
$$

(ii) The isometry orbit closure $\Sigma_{\mathcal{E}}(X)$ is the closure of $\mathcal{O}_{\mathcal{E}}(X)$ in the natural topology, i.e.,

$$
\Sigma_{\mathcal{E}}(X):=\overline{\mathcal{O}_{\mathcal{E}}(X)}
$$

Since $\mathcal{O}_{\mathcal{E}}(X) \subseteq \mathcal{D}_{r, R}$, the set $\Sigma_{\mathcal{E}}(X)$ is a compact set in the natural topology. Thus we obtain a topological dynamical system $\left(\Sigma_{\mathcal{E}}(X), \mathcal{E}^{n}\right)$ with an $\mathcal{E}^{n}$-action.

Now suppose that $X$ is a Delone set of isometry-finite type. For these sets the closure process yielding $\Sigma_{\mathcal{E}}(X)$ introduces no new $T$-neighborhoods, for any $T>0$.

Lemma 4.1. If $X$ is a Delone set of isometry-finite type, then

$$
\Sigma_{\mathcal{E}}(X)=\overline{[[X]]_{\mathcal{E}}}=\bigcup_{[[Y]]_{\mathcal{E}} \leq \mathcal{E}[[X]]_{\mathcal{E}}}[[Y]]_{\mathcal{E}} .
$$

Proof. By definition $\Sigma_{\mathcal{E}}(X)=\overline{[[X]]_{\mathcal{E}}}$, because any element $Y$ of $\Sigma_{\mathcal{E}}(X)$ is the limit in the natural topology of isometric copies of larger and larger stars of $X$, and all these $T$-stars occur in any element of $[[X]]_{\mathcal{E}}$. Any $T$-star in $Y$ is isometric to some $T$-star in $X$, because there are only finitely many $T$-stars up to isometry, so in any Cauchy sequence in 
the natural topology the approximating $T$-star becomes constant, up to isometry, hence the limit $T$-star is isometric to a $T$-star of $X$. Thus

$$
\Sigma_{\mathcal{E}}(X) \subseteq \bigcup_{[[Y]]_{\mathcal{E}} \leq \mathcal{E}[[X]]_{\mathcal{E}}}[[Y]]_{\mathcal{E}}
$$

Conversely any element in such $[[Y]]_{\mathcal{E}}$ with $[[Y]]_{\mathcal{E}} \leq_{\mathcal{E}}[[X]]_{\mathcal{E}}$ is the limit in the natural topology of isometric copies of larger and larger patches of $X$, hence $[[Y]]_{\mathcal{E}} \subseteq \Sigma_{\mathcal{E}}(X)$, so (4.4) follows.

Definition 4.3. The topological dynamical system $\left(\Sigma_{\mathcal{E}}(X), \mathcal{E}^{n}\right)$ is minimal under isometries if every $\mathcal{E}^{n}$-orbit of $\Sigma_{\mathcal{E}}(X)$ is dense.

We prove the following characterization of isometry-minimality.

Theorem 4.1. If $X$ is a Delone set of isometry-finite type, then the following conditions are equivalent:

(i) $[[X]]_{\mathcal{E}}$ is isometry-minimal.

(ii) The topological dynamical system $\left(\Sigma_{\mathcal{E}}(X), \mathcal{E}^{n}\right)$ is minimal, i.e., $[[X]]_{\mathcal{E}}$ is a closed set.

(iii) $X$ is isometry-repetitive. That is, for each $T$-star $\mathcal{P}$ of $X$ the set $X_{\mathcal{E}}(\mathcal{P})$ defined by

$$
X_{\mathcal{E}}(\mathcal{P}):=\left\{\mathbf{x}^{\prime} \in X: \mathcal{P}_{X}\left(\mathbf{x}^{\prime} ; T\right)=\varphi(\mathcal{P}) \text { for some isometry } \varphi\right\}
$$

is relatively dense.

Proof. (i) $\Leftrightarrow$ (ii) By Lemma $4.1[[X]]_{\mathcal{E}}$ is isometry-minimal means that $\Sigma_{\mathcal{E}}(X)=$ $[[X]]_{\mathcal{E}}$. For any $X^{\prime} \in[[X]]_{\mathcal{E}}$ we have by definition $\Sigma_{\mathcal{E}}\left(X^{\prime}\right)=\Sigma_{\mathcal{E}}(X)$, hence every orbit closure $\Sigma_{\mathcal{E}}\left(X^{\prime}\right)$ is the whole space. If to the contrary $\Sigma_{\mathcal{E}}(X) \neq[[X]]_{\mathcal{E}}$, then by Lemma 4.1 there is some $\left[\left[X^{\prime}\right]\right]_{\mathcal{E}}<_{\mathcal{E}}[[X]]_{\mathcal{E}}$, so that

$$
\Sigma_{\mathcal{E}}\left(X^{\prime}\right)=\bigcup_{[[Y]]_{\mathcal{E}} \leq \mathcal{E}\left[\left[X^{\prime}\right]\right]_{\mathcal{E}}}[[Y]]_{\mathcal{E}} \varsubsetneqq \Sigma_{\mathcal{E}}(X),
$$

hence $X^{\prime}$ does not have a dense orbit in $\left(\Sigma_{\mathcal{E}}(X), \mathcal{E}^{n}\right)$.

(i) $\Rightarrow$ (iii) We prove the contrapositive. If for some $T$-star $\mathcal{P}$ there are no isometric copies of $\mathcal{P}$ in arbitrarily large regions of $X$, say in $\left(X-\mathbf{x}_{i}\right) \cap B\left(0 ; T_{i}\right)$ with $T_{i} \rightarrow \infty$ as $i \rightarrow \infty$, we can translate these regions to the origin and extract a subsequence that converges in the natural topology to a limit set $Y$ that contains no isometric copy of $\mathcal{P}$. Thus $[[Y]]_{\mathcal{E}}<_{\mathcal{E}}[[X]]_{\mathcal{E}}$, so $[[X]]_{\mathcal{E}}$ is not minimal under isometries.

(ii) $\Rightarrow$ (i) Given a $T$-star $\mathcal{P}$ in $X$, by (iii) there is a radius $T^{\prime}$ such that every $T^{\prime}$-star of $X$ contains an isometric copy of $\mathcal{P}$. Thus given any $Y \in \overline{[[X]]_{\mathcal{E}}}$, since each $T^{\prime}$-patch in $Y$ is isometric to a $T^{\prime}$-patch of $X$, it contains an isometric copy of $\mathcal{P}$. This holds for all $\mathcal{P}$, so $Y \in[[X]]_{\mathcal{E}}$. Thus $\Sigma_{\mathcal{E}}(X)={\overline{[[X]]_{\mathcal{E}}}}_{\mathcal{B}}=[[X]]_{\mathcal{E}}$, which is therefore isometryminimal. 
Our next object is to derive several equivalent conditions for minimality under translations.

Definition 4.4. (i) A Delone set $X$ is repetitive if for each radius $T>0$ there is a finite value $M_{X}(T)$ such that each $M_{X}(T)$-neighborhood of $X$ contains a translate of each $T$-patch of $X$.

(ii) The repetitivity function $M_{X}(T)$ of $X$ is the smallest such value for each $T>0$.

A Delone set $X$ is repetitive if and only if it is a Delone set of finite type, such that the set of occurrences of each $T$-patch in $X$ is relatively dense.

We next construct a topological dynamical system with an $\mathbb{R}^{n}$-action associated to a given Delone set $X$, analogous to the construction in [50].

Definition 4.5. (i) Given a Delone set $X$, let $\mathcal{O}_{\mathcal{T}}(X)$ denote the orbit of $X$ under translations $\mathcal{T}^{n}$, i.e.,

$$
\mathcal{O}_{\mathcal{T}}(X):=\left\{X+\mathbf{t}: t \in \mathbb{R}^{n}\right\} .
$$

(ii) The translation orbit closure $\Sigma_{\mathcal{T}}(X)$ is the closure of $\mathcal{O}_{\mathcal{T}}(X)$ in the natural topology, i.e.,

$$
\Sigma_{\mathcal{T}}(X):=\overline{\mathcal{O}_{\mathcal{T}}(X)}
$$

We obtain a topological dynamical system $\left(\Sigma_{\mathcal{T}}(X), \mathbb{R}^{n}\right)$ with an $\mathbb{R}^{n}$-action.

Lemma 4.2. If $X$ is a Delone set of finite type, then

$$
\Sigma_{\mathcal{T}}(X)=\overline{[[X]]_{\mathcal{T}}}=\bigcup_{[[Y]]_{\mathcal{T}} \leq \mathcal{T}[[X]]_{\mathcal{T}}}[[Y]]_{\mathcal{T}} .
$$

Proof. Similar to Lemma 4.1.

Definition 4.6. The topological dynamical system $\left(\Sigma_{\mathcal{T}}(X), \mathbb{R}^{n}\right)$ is minimal if every $\mathbb{R}^{n}$-orbit of $\Sigma_{\mathcal{T}}(X)$ is dense.

Theorem 4.2. If $X$ is a Delone set of finite type in $\mathbb{R}^{n}$, then the following conditions are equivalent:

(i) $[[X]]_{\mathcal{T}}$ is minimal.

(ii) The topological dynamical system $\left(\Sigma_{\mathcal{T}}(X), \mathbb{R}^{n}\right)$ is minimal.

(iii) $X$ is repetitive. That is, for each centered T-patch $\mathcal{P}$ of $X$, the set $X_{\mathcal{T}}(\mathcal{P})$ defined by

$$
X_{\mathcal{T}}(\mathcal{P})=\left\{\mathbf{x}^{\prime} \in X: \mathcal{P}_{X}(\mathbf{x} ; T)=\mathcal{P}+\mathbf{t} \text { for some } \mathbf{t} \in \mathbb{R}^{n}\right\}
$$

is relatively dense.

Proof. Similar to Theorem 4.1. 
We next relate minimality under translations to minimality under isometries, as follows.

Theorem 4.3. If $X$ is a Delone set of finite type, then $[[X]]_{\mathcal{E}}$ is isometry-minimal if and only if $[[X]]_{\mathcal{T}}$ is minimal.

Proof. Since $X$ is a Delone set of finite type, Lemma 2.3 gives

$$
[[X]]_{\mathcal{E}}=O(n, \mathbb{R})[[X]]_{\mathcal{T}} .
$$

Suppose first that $[[X]]_{\mathcal{T}}$ is minimal, so that $[[X]]_{\mathcal{T}}$ is a closed set in the natural topology. To show that $[[X]]_{\mathcal{E}}$ is isometry-minimal we check that it is closed in the natural topology. So suppose that

$$
Y=\lim _{j \rightarrow \infty} Q_{j}\left(X-\mathbf{x}_{j}\right)
$$

with $Q_{j} \in O(n, \mathbb{R})$. We can extract a subsequence with $Q_{j_{k}} \rightarrow Q$, and

$$
Y=\lim _{k \rightarrow \infty} Q_{j_{k}}\left(X-\mathbf{x}_{j_{k}}\right)=\lim _{k \rightarrow \infty} Q\left(X-\mathbf{x}_{j_{k}}\right),
$$

using the Delone property of $X$. Thus

$$
\tilde{X}=Q^{-1} Y=\lim _{k \rightarrow \infty}\left(X-\mathbf{x}_{j_{k}}\right) \in[[X]]_{\mathcal{T}},
$$

because $[[X]]_{\mathcal{T}}$ is closed. Now $Y \in[[X]]_{\mathcal{E}}$ by (4.9).

Now suppose that $[[X]]_{\mathcal{E}}$ is isometry-minimal, so that it is a closed set in the natural topology. If $[[X]]_{\mathcal{T}}$ is not minimal, then there is some minimal set $\left[\left[X^{\prime}\right]\right]_{\mathcal{T}}$ contained in $\overline{[[X]}_{\mathcal{T}}$. (This is a standard fact in topological dynamics, proved by Zorn's lemma.) Now the set $X^{\prime} \in[[X]]_{\mathcal{E}}$ is dense in $[[X]]_{\mathcal{E}}$ and Lemma 2.3 gives

$$
[[X]]_{\mathcal{T}} \subseteq[[X]]_{\mathcal{E}}=\left[\left[X^{\prime}\right]\right]_{\mathcal{E}}=O(n, \mathbb{R})\left[\left[X^{\prime}\right]\right]_{\mathcal{T}} .
$$

There is some subset $G \subseteq O(n, \mathbb{R})$ such that $[[X]]_{\mathcal{T}}=G\left[\left[X^{\prime}\right]\right]_{\mathcal{T}}$. Consider a patch $\mathcal{P}$ of $X^{\prime}$ of radius $R$, which is therefore an enclosing set. Each image $\{g(\mathcal{P}): g \in G\}$ must be a patch of $X$, and if $g \neq g^{\prime}$, then the action $g$ and $g^{\prime}$ on the points of the patch $\mathcal{P}$ cannot be identical because $\mathcal{P}$ is an enclosing set. Any Delone set of finite type contains only finitely many translation-inequivalent $T$-patches for any $T$, see Theorem 2.2 of [34]. It follows that $G$ is a finite set. Thus

$$
[[X]]_{\mathcal{T}}=\bigcup_{i=1}^{|G|} g_{i}\left[\left[X^{\prime}\right]\right]_{\mathcal{T}}
$$

is a closed set in the natural topology, since $\left[\left[X^{\prime}\right]\right]_{\mathcal{T}}$ is closed. Now $[[X]]_{\mathcal{T}}$ is minimal by Theorem 4.1. (It follows that $[[X]]_{\mathcal{T}}=\left[\left[X^{\prime}\right]\right]_{\mathcal{T}}$ and $|G|=1$.)

We now turn to the proof of Theorem 1.1, which is based on constructing a watermark with $G=P \operatorname{sym}\left([[X]]_{\mathcal{T}}\right)$. We need a preliminary lemma. If $\mathcal{P}$ is a centered $\rho$-patch of a set $X$, let

$$
\operatorname{Psym}(\mathcal{P}):=\left\{Q \in O(n, \mathbb{R}): Q \mathcal{P} \in \mathcal{A}_{X}(\rho)\right\}
$$


We always have

$$
\operatorname{Psym}\left([[X]]_{\mathcal{T}}\right) \subseteq \operatorname{Psym}(\mathcal{P}) .
$$

The following lemma gives conditions where equality occurs.

Lemma 4.3. Let $X$ be a Delone set of finite type in $\mathbb{R}^{n}$ which is minimal. There is a radius $\rho_{0}$ depending on $X$, with $\rho_{0} \geq R$, such that if $\rho \geq \rho_{0}$, then every $\rho$-patch $\mathcal{P}$ satisfies

$$
\operatorname{Psym}(\mathcal{P})=\operatorname{Psym}\left([[X]]_{\mathcal{T}}\right)
$$

Proof. If $\rho \geq R$, then every centered $\rho$-patch is an enclosing set of $\mathbf{0}$, hence $\operatorname{Psym}(\mathcal{P})$ is a finite group. We first show there is no group of rotations $G^{\prime}$ that strictly contains $G$ such that

$$
G^{\prime} \subseteq \operatorname{Psym}(\mathcal{P}) \quad \text { for all patches } \mathcal{P} \text {. }
$$

Suppose there were. Now $G^{\prime}$ must be finite, and (4.13) would imply that for each $g^{\prime} \in G^{\prime}$ and $X^{\prime} \in[[X]]_{\mathcal{T}}$ one has $g^{\prime} X^{\prime} \in[[X]]_{\mathcal{T}}$, because every patch of $g^{\prime} X^{\prime}$ is translationequivalent to a patch of $X$ by (4.10) and (4.13). Thus $G^{\prime} \subseteq P \operatorname{sym}\left([[X]]_{\mathcal{T}}\right)$, which is a contradiction. It follows that we have

$$
\bigcap_{\mathcal{P}} \operatorname{Psym}(\mathcal{P})=\operatorname{Psym}\left([[X]]_{\mathcal{T}}\right) .
$$

Now take any fixed patch $\mathcal{P}_{0}$ of radius $R$ and since $\operatorname{Psym}\left(\mathcal{P}_{0}\right)$ is a finite group, we can find a finite set of patches $\mathcal{P}_{1}, \ldots, \mathcal{P}_{k}$, where $\mathcal{P}_{i}$ is a $\rho_{i}$-patch with $\rho_{i} \geq R$, such that if $g^{\prime} \in \operatorname{Psym}\left(\mathcal{P}_{0}\right) \backslash G$, then $g^{\prime} \notin \operatorname{Psym}\left(\mathcal{P}_{i}\right)$ for some $i$ with $1 \leq i \leq k$. All these patches, including $\mathcal{P}_{0}$, are of radius at most

$$
\rho^{\prime}:=\max \left\{\rho_{i}: 1 \leq i \leq k\right\} .
$$

Since $X$ is minimal, by Theorem 4.2(iii) it follows for any $\rho \geq M_{X}\left(\rho^{\prime}\right)$ that any $\rho$-patch $\mathcal{P}$ contains a translate of each of the patches $\mathcal{P}_{0}, \mathcal{P}_{1}, \ldots, \mathcal{P}_{k}$. Now (4.12) holds for such a patch $\mathcal{P}$, because any rotation that maps $\mathcal{P}$ to a legal $\rho$-patch must simultaneously map each translate of $\mathcal{P}_{i}$ to a translate of a similarly rotated legal $\rho_{i}$-patch.

Proof of Theorem 1.1. We are given a Delone set $X$ of finite type such that $X$ is minimal. Our object is to show that, for any sufficiently large value of $\rho$, the set $\mathcal{L}=\mathcal{L}_{\rho}$ of all translation-equivalence classes of $\rho$-patches of $X$ has a watermark $(\Delta, G)$, in which $G=\operatorname{Psym}\left([[X]]_{\mathcal{T}}\right)$. If so, then Theorem 1.1 will follow from Theorem 3.1.

We apply Lemma 4.3, and take for $\Delta$ any centered patch $\mathcal{P}$ for which (4.12) holds. Let it be of radius $T$, with $T \geq R$, and then $\mathcal{P}$ is $R$-enclosing, hence $T$-enclosing. We claim that the pair $(\Delta, G)$ is a watermark for the set $\mathcal{L}=\mathcal{L}_{\rho}$ of all $\rho$-patches with

$$
\rho:=2 M_{X}(T)
$$

Now $M_{X}(T) \geq T$ trivially, hence we have $T \leq \frac{1}{2} \rho$, so property (i) of Definition 3.2 holds. Property (ii) of Definition 3.2 holds by definition of the repetitivity function. 
Property (iii) of Definition 3.2 holds by (4.12). Property (iv) of Definition 3.2 holds because, by definition of $M_{X}(T)$, the set of centers of translated copies of any $T$-patch is relatively dense with constant at most $M_{X}(T)$, so that any $2 M_{X}(T)$-patch centered at a point $\mathbf{x} \in X$ which is the center of $\Delta$ has translates that are strongly $M_{X}(T)$-enclosing, by Lemma 2.1(ii).

\section{Perfect Local Rules}

Most of the explicit constructions of quasicrystalline structures by "local rules" in the literature involve perfect local rules.

Definition 5.1. (i) A set of local rules $\mathcal{L}$ are perfect local rules under isometries if all $X$ that satisfy $\mathcal{L}$ are Delone sets of isometry-finite type that fall in a single local isomorphism class $[[X]]_{\mathcal{E}}$ under isometries, which is necessarily isometry-minimal. That is,

$$
\Sigma_{\mathcal{E}}(\mathcal{L})=[[X]]_{\mathcal{E}}
$$

We say that $X$ has perfect local rules under isometries if $[[X]]_{\mathcal{E}}$ does.

(ii) A set of local rules $\mathcal{L}$ are perfect local rules under translations if all $X$ that satisfy $\mathcal{L}$ under translations are Delone sets of finite type and fall in single local isomorphism class $[[X]]_{\mathcal{T}}$, which is necessarily minimal. That is,

$$
\Sigma_{\mathcal{T}}(\mathcal{L})=[[X]]_{\mathcal{T}}
$$

We say that $X$ has perfect local rules under translations if $[[X]]_{\mathcal{T}}$ does.

The main result of this section is to show that for Delone sets of finite type these two concepts of perfect local rules coincide. We prove the two implications separately.

Theorem 5.1. Let $X$ be a Delone set of finite type in $\mathbb{R}^{n}$. If $X$ has perfect local rules under isometries, then $X$ has perfect local rules under translations.

Proof. Since $X$ has perfect local rules under isometries, it is isometry-minimal. Because $X$ is a Delone set of finite type, it is minimal by Theorem 4.3.

Suppose that there is a set $\mathcal{L}$ of perfect local rules under isometries for $X$ of radius $T$. We let $\mathcal{L}_{\rho}$ denote the set of centered $\rho$-patches of $X$, which is a finite set because $X$ is of finite type. Now Lemma 2.3 gives

$$
[[X]]_{\mathcal{E}}=O(n, \mathbb{R})[[X]]_{\mathcal{T}} .
$$

If $\rho \geq T$, then any set $X^{\prime}$ that satisfies $\mathcal{L}_{\rho}$ under translations also satisfies $\mathcal{L}$ under isometries. Thus it is contained in $[[X]]_{\mathcal{E}}$, hence is of the form

$$
X^{\prime}=Q \tilde{X} \quad \text { for some } \quad Q \in O(n, \mathbb{R}) \quad \text { and } \quad \tilde{X} \in[[X]]_{\mathcal{T}} .
$$

By translating $X^{\prime}$ and $\tilde{X}$ we may reduce to the case that $\mathbf{0} \in X^{\prime}$. Since $\rho \geq T \geq R$, the patch $\mathcal{P}=\mathcal{P}(\mathbf{0} ; \rho)$ of $X^{\prime}$ strongly $R$-encloses $\mathbf{0}$, and by hypothesis is contained in $\mathcal{L}_{\rho}$. 
Now (5.4) yields

$$
Q \subseteq \operatorname{Psym}(\mathcal{P})
$$

which is a finite group. Since $X$ is minimal, by Lemma 4.3 there is a constant $\rho_{0}$ depending on $X$ such that, for all $\rho \geq \rho_{0}$, every $\rho$-patch $\mathcal{P}$ has

$$
\operatorname{Psym}(\mathcal{P})=\operatorname{Psym}\left([[X]]_{\mathcal{T}}\right) .
$$

Now (5.3) gives for $\rho \geq \max \left(\rho_{0}, T\right)$ that

$$
X^{\prime} \in \operatorname{Psym}\left([[X]]_{\mathcal{T}}\right)[[X]]_{\mathcal{T}}=[[X]]_{\mathcal{T}},
$$

hence $\mathcal{L}_{\rho}$ is a set of perfect local rules under translations.

It seems reasonable to conjecture that if a set $X$ has perfect local rules under isometries of radius $\rho$, then it has perfect local rules under translations for the same radius $\rho$.

We now prove a converse result.

Theorem 5.2. Let $X$ be a Delone set of finite type in $\mathbb{R}^{n}$. If $X$ has perfect local rules under translations, then it has perfect local rules under isometries.

Proof. We prove this result using a watermark $(\Delta, G)$, where $G=P \operatorname{Psym}\left([[X]]_{\mathcal{T}}\right)$. Let $X$ have perfect local rules under translations of radius $T$, say, and note that $X$ is minimal. Then by Lemma 4.3 there exists a constant $\rho_{0} \geq 2 R$ such that for all $\rho \geq \rho_{0}$ every $\rho$-patch $\mathcal{P}$ of $X$ has $P \operatorname{sym}(\mathcal{P})=G$. Let $\mathcal{L}$ consist of all $\rho_{1}$-patches of $X$ with

$$
\rho_{1}:=\max \left(2 M_{X}\left(\rho_{0}\right), T\right) .
$$

Here $\rho_{1}$ is finite since $X$ is minimal.

We will show that $\mathcal{L}$ is a set of perfect local rules under isometries for $X$. To prove this, let $\Delta$ be a fixed centered $\rho_{0}$-patch of $X$. Since $\rho_{1} \geq 2 M_{X}\left(\rho_{0}\right)$, the set $\mathcal{L}$ contains $(\Delta, G)$ as a watermark. The watermark property is established by the same proof as in Theorem 1.1.

Suppose next that $Z \in \Sigma_{\mathcal{E}}(\mathcal{L})$. Then $Z$ contains an isometric copy $\tilde{\Delta}$ of $\Delta$, so take an isometry $\tilde{Z}=Q Z+\mathbf{t}$ that moves $\tilde{\Delta}$ to $\Delta$. Now by definition of $M_{X}\left(\rho_{0}\right)$ the set of centers of isometric copies $\tilde{\Delta}$ of $\Delta$ in any $\Delta$-centered element of $\mathcal{L}$ form an $M_{X}\left(\rho_{0}\right)$-enclosing set of $\mathbf{0}$. We now claim that every isometric copy $\tilde{\Delta}$ of $\Delta$ in $\tilde{Z}$ satisfies

$$
\tilde{\Delta}=Q \Delta+\mathbf{t}, \quad \text { for some } \quad Q \in G \quad \text { and } \quad \mathbf{t} \in \mathbb{R}^{n} .
$$

We prove this claim by an inductive proof similar to that used in the proof of Theorem 3.1. Each point $\tilde{\mathbf{z}} \in \tilde{Z}$ that is a center of some $\tilde{\Delta}$ must have as a $\rho_{1}$-patch some $\tilde{Q} Y_{i}+\mathbf{t}$ for some $Y_{i} \in \mathcal{L}$. If this $\rho_{1}$-patch contains some region $\tilde{\Delta}^{*}$ that satisfies (5.6), then $\tilde{Q} Y_{i}$ must be correctly oriented to match the orientation of $\tilde{\Delta}^{*}$. Since $\tilde{\Delta}^{*}$ is an enclosing set, this forces $\tilde{Q} \in G$, hence $\tilde{Q} Y_{i} \in \mathcal{L}$ because the set of centered $\rho_{1}$-patches of $X$ is invariant under $\operatorname{Psym}\left([[X]]_{\mathcal{T}}\right)=G$. Thus (5.6) must hold for every isometric copy of $\Delta$ in this $\rho_{1}$-patch. By an induction starting from the original set $\Delta$ centered at 0 , we obtain by 
induction on lists $\mathcal{C}_{k}, \mathcal{M}_{k}$ that every isometric copy $\tilde{\Delta}$ of $\Delta$ in $\tilde{Z}$ satisfies (5.6), and furthermore the rules of $\mathcal{L}$ under isometries that were applied at points $\tilde{\mathbf{z}}$, were actually applied using translations. Since $X$ has perfect local rules under translations, and since $\rho_{1} \geq T$, this yields

$$
\tilde{Z} \in \Sigma_{\mathcal{T}}(\mathcal{L})=[[X]]_{\mathcal{T}}
$$

It follows that

$$
Z \in O(n, \mathbb{R}) \Sigma_{\mathcal{T}}(\mathcal{L}) \equiv O(n, \mathbb{R})[[X]]_{\mathcal{T}}
$$

By Lemma 2.3 this gives $Z \in[[X]]_{\mathcal{E}}$, hence $\Sigma_{\mathcal{E}}(\mathcal{L})=[[X]]_{\mathcal{E}}$.

Remark. To get an explicit bound on the radius $\rho_{1}$ for local rules under isometry needed in Theorem 5.2, an upper bound for the order of the group $G=\operatorname{Psym}\left([[X]]_{\mathcal{T}}\right)$ and upper bounds on the repetitivity function $M_{X}(\rho)$ are needed. An explicit bound for $\rho_{1}$ for the case of ideal crystals $X$ is given in [15], namely, $\rho_{1}=2 n^{2} \log _{2}(R / r+2) R k$, where $k$ is the number of points in a unit cell of $X$.

\section{Acknowledgments}

I am indebted to M. Baake, L. Danzer, M. Senechal, C. Radin, and the referees for helpful comments and references. In addition F. Gähler provided helpful comments and corrections to the Appendix.

\section{Appendix. Local Rules and Matching Rules for Tilings}

Much of the work on quasicrystalline structures has been formulated in terms of tiling models of quasicrystals. There is a considerable body of work on "local rules" and "matching rules" for tilings, see [42], [30], [31], and [57]. These concepts of "local rules" are intended to enforce specific properties in all the tilings that satisfy them: these properties may include being a Meyer set or cut-and-project set $^{6}$ or being in a single local isomorphism class (perfect local rules as defined in this paper). Below we give more precise equivalences. Much literature on local rules and matching rules of tilings requires that such rules enforce nonperiodicity of any structure $X$ that satisfies them, e.g., Definition 7.1 of [57]. We make no such requirement here.

We consider tilings of $\mathbb{R}^{n}$ by translations of a finite set $\mathcal{F}$ of prototiles, each of which is a convex polytope. For example, the Voronoi domains associated to the points of a Delone set of finite type give such a finite set $\mathcal{F}$ of prototiles, see Theorem 2.2 of [34]. To any such tiling $\mathcal{T}$ we associate the vertex set $V(\mathcal{T})$ which consists of all vertices of the polytopes in the tiling, and the centroid set $C(\mathcal{T})$ which consists of the centroids ${ }^{7}$ (centers of gravity) of all polytopes in the tiling. It is easy to show that $V(\mathcal{T})$ and $C(\mathcal{T})$ are always Delone sets of finite type.

\footnotetext{
${ }^{6}$ The term quasiperiodic tiling is used by some authors to mean that the tiling has a pure point component in their diffraction spectrum, whose support is contained in a $\mathbb{Z}$-module of finite rank, see [21] and [43]. However, the constructions given typically produce cut-and-project sets, which are known to have pure discrete spectrum.

${ }^{7}$ If the polytopes are centrally symmetric, the centroid is the center of symmetry. Thus for a Delone set of finite type $X$, we have $X=C(\mathcal{T})$ where $\mathcal{T}$ is the tiling given by its Voronoi domains.
} 
Much work in the literature is restricted to the special case in which the prototile set $\mathcal{F}$ consists of all zonotopes generated by all subsets of $n$ linearly independent vectors formed from a collection of $s$ vectors $\left\{\mathbf{v}_{1}, \mathbf{v}_{2}, \ldots, \mathbf{v}_{s}\right\}$ in $\mathbb{R}^{n}$. Assuming all these vectors are linearly independent, there are $\left(\begin{array}{l}n \\ s\end{array}\right)$ such zonotopes, given by

$$
P_{I}:=\left\{x_{1} \mathbf{v}_{i_{1}}+x_{2} \mathbf{v}_{i_{2}}+\cdots+x_{n} \mathbf{v}_{i_{n}}: 0 \leq x_{i} \leq 1\right\}, \quad \text { for } \quad I=\left\{i_{1}, \ldots, i_{n}\right\},
$$

where $I=\left\{i_{1}, i_{2}, \ldots, i_{n}\right\} \subseteq\{1,2, \ldots, s\}$. We call any tiling of $\mathbb{R}^{n}$ using such prototiles a zonotope tiling.

Several notions of local rules were introduced by Levitov [42] for zonotope tilings. A set of $r$-rules $\mathcal{L}$ consists of a finite list of patches of contiguous tiles, each of which fits completely inside a ball of radius $r$. A (zonotope) quasicrystal ${ }^{8}$ is any zonotope tiling $\mathcal{T}$, resulting from a cut-and-project construction from the lattice $\mathbb{Z}^{s}$, which is defined using a fixed linear embedding $L: \mathbb{R}^{n} \rightarrow \mathbb{R}^{s}$ which we call the supporting map for $\mathcal{T}$ and a vector $\mathbf{c} \in \mathbb{R}^{s}$ which we call the offset vector. Here $\mathcal{T}$ has vertex set $V(\mathcal{T})$ given by orthogonal projection of the vertices of the unit cubes in $\mathbb{Z}^{n}$ which intersect the translated subspace $L\left(\mathbb{R}^{n}\right)+\mathbf{c}$ onto $L\left(\mathbb{R}^{n}\right)$, and we identify $L\left(\mathbb{R}^{n}\right)$ with $\mathbb{R}^{n}$ using $L$. Levitov says that a zonotope tiling $\mathcal{T}$ has strong local rules if its set of $r$-rules $\mathcal{L}$ for some fixed $r$ has the following properties:

(i) $\mathcal{T}$ is a zonotope quasicrystal.

(ii) Every zonotope tiling that satisfies $\mathcal{L}$ is a zonotope quasicrystal with the same supporting map $L: \mathbb{R}^{n} \rightarrow \mathbb{R}^{s}$, but possibly a different offset vector.

This definition implies that the vertex set $V(\mathcal{T})$ has perfect local rules in the sense of Section 5, provided that the projection of the lattice generated by the zonotope vertices on internal space $\mathbb{R}^{s-n}$ is dense; otherwise there are uncountably many local isomorphism classes of zonotope tilings, depending on the offset vector.

Levitov [42] says that a zonotope tiling $\mathcal{T}$ has weak local rules if its set of $r$-rules $\mathcal{L}$ for some fixed $r$ has the following properties:

(i) $\mathcal{T}$ is a zonotope quasicrystal.

(ii) Every zonotope tiling that satisfies $\mathcal{L}$ has vertex set $V(\mathcal{T})$ that is a projection of a subset of $\mathbb{Z}^{n}$ onto $L\left(\mathbb{R}^{s}\right)+\mathbf{c}$ that consists only of points at most a constant distance from $L\left(\mathbb{R}^{s}\right)+\mathbf{c}$.

Condition (ii) says the vertex set $V\left(\mathcal{T}^{\prime}\right)$ of any tiling $\mathcal{T}^{\prime}$ that satisfies $\mathcal{L}$ is a Meyer set which has support function $L$, see Theorem 3.1 of [34]. Burkov [6] shows that a certain eight-fold symmetric parallelogram tiling of $\mathbb{R}^{2}$ does not have weak local rules in this sense. Ingersent [29] further refines this classification of local rules.

Le and Sadov (see p. 72 of [39]) introduce related concepts. Their definition of set of local rules based on atlases corresponds to a set of local rules under translations for the centroid set $C(\mathcal{T})$. They also define local rules based on colorings, which are a special case of matching rules, as defined below.

Matching rules enlarge the prototile set by replacing each prototile with a finite number of related prototiles, by adding marks or colors to the prototiles. Matching rules consist

\footnotetext{
${ }^{8}$ This is a special case of a quasiperiodic tiling as defined in [39], see also [43].
} 
of a finite list of ways that adjacent tiles with the markings can be legally arranged (under translations). Sometimes matching rules are prescribed as colorings of vertices or faces of the polyhedra, with a requirement that colorings on immediately adjacent tiles must match; these can be formulated as vertex colorings. These definitions have been formulated as restrictions on immediately adjacent tiles (as in a jigsaw puzzle) but we may obviously generalize them to allow a finite list of legal colorings of larger $r$-patches that include nonadjacent tiles. We call these generalized matching rules. The effect of matching rules is to narrow the class of allowed tilings.

A notion of equivalence of different tilings called mutual local derivability was introduced by Baake and Schlottmann [4], [3]. A tiling $\mathcal{T}_{2}$ is said to be locally derivable from a tiling $\mathcal{T}_{1}$ if there is an isometric ${ }^{9}$ copy $\mathcal{T}_{1}^{\prime}=Q \mathcal{T}_{1}+\mathbf{t}$ and if there is a radius $R^{\prime}$ such that the location of the tiles of $T_{2}$ in the ball $B(\mathbf{x}, 3 R)$ is entirely determined by the patch of $\mathcal{T}_{1}^{\prime}$ in the ball $B\left(\mathbf{x} ; R^{\prime}\right)$, where $R$ is the diameter of the largest tile in $\mathcal{T}_{2}$. Two tilings $\mathcal{T}_{1}$ and $\mathcal{T}_{2}$ are mutually locally derivable if each is locally derivable from the other. An $M L D$-class of tilings, denoted $M L D(\mathcal{T})$, is the set of all tilings mutually locally derivable from $\mathcal{T}$. It is a union of local isomorphism classes of tilings. If $\mathcal{T}$ is a tiling satisfying a set of matching rules, then the tiling $\mathcal{T}^{\prime}$ obtained from it by erasing the markings is locally derivable from $\mathcal{T}$, but the converse need not hold: they may be in different MLD classes.

We say that a tiling has perfect matching rules if there exists a set of matching rules such that all tilings that satisfy it are in the same local isomorphism class of tilings under translations. That is, the allowed tilings with the markings added have perfect local rules. If one tiling in an MLD class has perfect matching rules, then they all do, see Theorem 11.2 of [2].

We can define analogous notions for Delone sets (under translations or under isometries). A Delone set $X_{2}$ is locally derivable under isometries (resp.translations) from a Delone set $X_{1}$ if there is an isometric copy $X_{1}^{\prime}=Q X_{1}+\mathbf{t}$ (resp. translation $X_{1}+\mathbf{t}$ ) and a value $R^{\prime}$ such for each $\mathbf{x} \in X_{1}^{\prime}$ each $3 R$-neighborhood $X_{2} \cap B(\mathbf{x}, 3 R)$ is determined by the isometry equivalence class of the set $X_{1}^{\prime} \cap B\left(\mathbf{x}, R^{\prime}\right)$. We say that $X_{1}$ and $X_{2}$ are mutually locally derivable if each is locally derivable from the other (under isometries or translations, respectively).

Finding generalized matching rules using vertex colorings is analogous to changing the Delone set of finite type $X$ (under translations) by replacing it with a new Delone set $X^{\prime}$ in which each point $\mathbf{x}$ of $X$ is replaced with a particular finite local configuration of points $\mathcal{W}_{i}+\mathbf{x}$, where $\mathcal{W}_{i}$ is drawn from a finite list $\mathcal{L}^{\prime}$ of point configurations, each of which includes $\mathbf{0}$, and all are inside the ball $B\left(\mathbf{0}, \frac{1}{4} r\right)$, where $X$ is an $(r, R)$-set. The list $\mathcal{L}^{\prime}$ of configurations plays the role corresponding to the list of colors that might be assigned to vertex $\mathbf{x}$. In such a construction the resulting set $X^{\prime}$ always satisfies

$$
X \subseteq X^{\prime}
$$

In particular, we may choose each element of $\mathcal{W}_{i}$ to consist of $n+2$ points, which consist of $\mathbf{0}$ and the vertices of a simplex enclosing $\mathbf{0}$, with no two of these simplices equivalent

\footnotetext{
${ }^{9}$ Baake et al. [4] and Gähler et al. [22] also permit similarities $\mathcal{T}_{1} \rightarrow \lambda \mathcal{T}_{1}$ in this definition. We exclude similarities here.
} 
under isometries. In this case we can recover $X$ from $X^{\prime}$ as the set of centers of $\frac{1}{4} r$-stars of $X^{\prime}$ that are enclosing sets. Thus $X$ is locally derivable from $X^{\prime}$, but $X^{\prime}$ may not be locally derivable from $X$.

We can use the definitions above to define a notion of perfect matching rules for Delone sets, as follows.

Definition A.1. A Delone set $X$ has perfect matching rules under isometries (resp. under translations) if it is locally derivable under isometries (resp. under translations) from a Delone set $X^{\prime}$ which has perfect local rules under isometries (resp. under translations).

Nontrivial sets $X$ which have perfect local rules (under translations) can be obtained from translation tilings of $\mathbb{R}^{n}$ with a finite number of tiles that have "perfect matching rules." A different finite set of points is assigned to each prototile with the matching conditions added, and $X$ is the set of points that results from a tiling that obeys the "perfect matching rules." Such a conversion method is described in specific cases in [32] and [22]. A general method for proving that certain tilings have "perfect matching rules" is sketched in [20]. It is based on tile sets having an inflation-deflation rule having the unique composition property, which appears in special cases in [1], see also [59]. In particular, it has been repeatedly demonstrated that Penrose tilings have "perfect matching rules" under translations. A three-dimensional generalization appears in [10].

In a similar fashion tilings with perfect local rules under isometries can be converted to Delone sets having perfect local rules under isometries. The most general result demonstrating perfect local rules under isometries for tilings is that of Goodman-Straus [24], which proves that perfect matching rules exist for nearly all substitution tilings, i.e., perfect local rules after the matching conditions are added.

\section{References}

1. R. Ammann, B. Grünbaum, and G. C. Shephard, Aperiodic tiles, Discrete Comput. Geom. 8 (1992), 1-25.

2. M. Baake, A guide to mathematical quasicrystals, Preprint.

3. M. Baake and M. Schlottmann, Geometric aspects of tilings and equivalence concepts, in: Proc. 5th Internat. Conf. on Quasicrystals (C. Janot and R. Mossieri, Eds.), World Scientific: Singapore, 1995, pp. $15-21$.

4. M. Baake, M. Schlottmann, and P. D. Jarvis, Quasiperiodic tilings with tenfold symmetry and equivalence with respect to local derivability, J. Phys. A 24 (1991), 4637-4654.

5. D. Berend and C. Radin, Are there chaotic tilings?, Comm. Math. Phys. 152 (1993), 215-219.

6. S. E. Burkov, Absence of weak local rules for the planar quasicrystalline tiling with the 8-fold rotational symmetry, Comm. Math. Phys. 119 (1988), 667-675.

7. S. E. Burkov, Modelling decagonal quasicrystals: random assembly of interpenetrating decagonal clusters, J. Phys. France 2 (1992), 695-706.

8. S. E. Burkov, Enforcement of matching rules by chemical ordering in the decagonal Al Cu Co quasicrystal, Phys. Rev. B 47 (1993), 12325-12328.

9. J. H. Conway and C. Radin, Quaquaversal tilings and rotations, Invent. Math. 132 (1998), 179-188.

10. L. Danzer, Three dimensional analogs of planar Penrose tilings and quasicrystals, Discrete Math. 76 (1989), 1-7.

11. L. Danzer, Quasiperiodicity: local and global aspects, in: Group-Theoretical Methods in Physics (V. V. Dodonov and V. I. Man'ko, Eds.), Lecture Notes in Physics, Vol. 382, Springer-Verlag: New York, 1991, pp. 561-572. 
12. L. Danzer, A family of 3D-spacefillers not permitting any periodic or quasiperiodic tiling, Proc. APERIODIC'94 (1995), pp. 11-17.

13. L. Danzer and N. P. Dolbilin, Delone graphs: some species and local rules, in: The Mathematics of Long-Range Aperiodic Order (R. V. Moody, Ed.), Kluwer: Dordrecht, 1997, pp. 85-114.

14. B. N. Delone, N. P. Dolbilin, M. I. Shtogrin and R. V. Galiulin, A local criterion for regularity of a system of points, Soviet Math. Dokl. 17(2) (1976), 319-322.

15. N. P. Dolbilin, J. C. Lagarias, and M. Senechal, Multiregular point systems, Discrete Comput. Geom. 20 (1998), 477-498.

16. V. Elser, Comment on "quasicrystals": a new class of ordered structures, Phys. Rev. Lett. 85 (1984), 1509-1513.

17. H. Furstenberg, Disjointness in ergodic theory, minimal sets, and a problem in Diophantine approximation, Math. Systems Theory 1 (1967), 1-49.

18. H. Furstenberg, Recurrence in Ergodic Theory and Combinatorial Number Theory, Princeton University Press: Princeton, NJ, 1981.

19. F. Gähler, Some mathematical problems arising in the study of quasicrystals. J. Phys. Colloq. 47(3) (1986), $115-123$.

20. F. Gähler, Matching rules for quasicrystals: the composition-decomposition method, J. Noncryst. Solids 153 \& 154 (1993), 160-164.

21. F. Gähler, Self-affine quasiperiodic tilings, in: GROUP 21: Physical Applications and Mathematical Aspects of Geometry, Groups and Algebras, Vol. 2 (H.-D. Doebner, W. Scherer, and C. Schulte, Eds.), World Scientific: Singapore, 1997, pp. 972-976.

22. F. Gähler, M. Baake, and M. Schlottman, Binary tiling quasicrystals and matching rules, Phys. Rev. B 50 (1994) 12458-12467.

23. A. I. Goldman, J. W. Anderegg, M. F. Besser, S.-L. Chang, D. W. Delaney, C. J. Jenks, M. J. Kramer, T. A. Lograsso, D. W. Lynch, R. W. McCallum, J. E. Shield, D. J. Sordelet, and P. A. Thiel, Quasicrystalline Materials, Amer. Sci. 84 (1996), 230-241.

24. C. Goodman-Strauss, Matching rules and substitution tilings, Ann. of Math. 147 (1998), 181-223.

25. B. Grünbaum and G. C. Shephard, Tilings and Patterns, Freeman: San Francisco, CA, 1987.

26. C. Henley, Random tilings with quasicrystal order: transfer-matrix approach, J. Phys. A 21 (1988), 1649_ 1677.

27. C. L. Henley, Random tiling models, in: Quasicrystals: The State of the Art (D. P. DiVincenzo and P. J. Steinhardt, Eds.), World Scientific: Singapore, 1991, pp. 429-524.

28. A. Hof, Diffraction by aperiodic structures, in: The Mathematics of Long-Range Aperiodic Order (R. V. Moody, Ed.), Kluwer: Dordrecht, 1997, pp. 239-268.

29. K. Ingersent, Matching rules for quasicrystalline tilings, in: Quasicrystals-The State of the Art (D. P. DiVincenzo and P. J. Steinhardt, Eds.), World Scientific: Singapore, 1991, pp. 185-212.

30. A. Katz, Theory of matching rules for three-dimensional Penrose tilings, Comm. Math. Phys. 188 (1988), 263-288.

31. A. Katz, M. Duneau, and C. Oguey, A geometric approach to quasiperiodic tilings, Comm. Math. Phys. 118 (1988), 99-118.

32. R. Klitzing and M. Baake, Representation of certain self-similar quasiperiodic tilings with perfect matching rules by discrete point sets, J. Phys. France 4 (1994), 893-904.

33. J. C. Lagarias, Meyer's concept of quasicrystal and quasiregular sets, Comm. Math. Phys. 179 (1996), 365-376.

34. J. C. Lagarias, Geometric models for quasicrystals, I. Delone sets of finite type, Discrete Comput. Geom. 21 (1999), 161-191.

35. J. C. Lagarias and P. A. B. Pleasants, Repetitive Delone sets and perfect quasicrystals, Preprint.

36. T. Q. T. Le, Local rules for pentagonal quasicrystals, Discrete Comput. Geom. 14 (1995), 31-70.

37. T. Q. T. Le, Local rules for multidimensional quasicrystals, Differential Geom. Appl. 5 (1995), 13-31.

38. T. Q. T. Le, Local rules for quasiperiodic tilings, in: The Mathematics of Long Range Aperiodic Order (R. V. Moody, Ed.), Kluwer: Dordrecht, 1997, pp. 331-366.

39. T. Q. T. Le, S. A. Piunikhin, and V. A. Sadov, The geometry of quasicrystals, Russian Math. Surveys $\mathbf{4 8}(1)$ (1993), 37-100.

40. D. Levine and P. J. Steinhardt, Quasicrystals: a new class of ordered structures, Phys. Rev. Lett. 53 (1984), 2477-2480. 
41. D. Levine and P. J. Steinhardt, Quasicrystals, I. Definition and structure, Phys. Rev. B 34 (1986), 596-615.

42. L. S. Levitov, Local rules for quasicrystals, Comm. Math. Phys. 119 (1988), 627-666.

43. W. F. Lunnon and P. A. B. Pleasants, Quasicrystallographic tilings, J. Math. Pures Appl. 66 (1987), 217-263.

44. N. D. Mermin, Quasicrystallography is better in Fourier space, in: Quasicrystals: The State of the Art (D. P. DiVincenzo and P. J. Steinhardt, Eds.), World Scientific: Singapore, 1991, pp. 133-183.

45. R. V. Moody, Meyer sets and the finite generation of quasicrystals, in: Symmetries in Science VIII (P. Gruber, Ed.), Plenum: London, 1995, pp. 379-394.

46. R. V. Moody, Meyer sets and their duals, in: The Mathematics of Long-Range Aperiodic Order (R. V. Moody, Ed.), Kluwer: Dordrecht, 1997, pp. 403-442.

47. S. A. Piunikhin, Quasicrystallographic groups in the sense of Novikov, Math. Zametki 47(5) (1990), 81-87 [Math. Notes 47 (1990), 478-482].

48. C. Radin, Correlations in classical ground states, J. Statist. Phys. 43 (1986), 707-712.

49. C. Radin, Low temperature and the origin of crystalline symmetry, Internat. J. Mod. Phys. B 1 (1987), 1157-1191.

50. C. Radin, Global order from local sources, Bull. Amer. Math. Soc. 25 (1991), 335-364.

51. C. Radin, $\mathbf{Z}^{n}$ versus $\mathbf{Z}$ Actions for Systems of Finite Type, Contemporary Mathematics, Vol. 135, American Mathematical Society: Providence, RI, 1992, pp. 339-342.

52. C. Radin, The pinwheel tilings of the plane, Ann. of Math. 139 (1994), 661-702.

53. C. Radin, Space tilings and substitutions, Geom. Dedicata 55 (1995), 257-264.

54. C. Radin and M. Wolff, Space tilings and local isomorphism, Geom. Dedicata 42 (1992), 355-360.

55. E. A. Robinson, Jr., The dynamical theory of tilings and quasicrystallography, in: Ergodic Theory of $\mathbb{Z}^{d}$ Actions (M. Pollicott and K. Schmidt, Eds.), London Mathematical Society Lecture Notes, Vol. 228, Cambridge University Press: Cambridge, 1996, pp. 451-473.

56. R. L. E. Schwartzenberger, N-Dimensional Crystallography, Pitman: London, 1980.

57. M. Senechal, Quasicrystals and Geometry, Cambridge University Press: Cambridge, 1995.

58. B. Simon, Fifteen problems in mathematical physics, in: Perspectives in Mathematics, Birkhaüser Verlag: Basel, 1984, pp. 423-454.

59. B. Solomyak, Non-periodicity implies unique composition for self-similar translationally-finite tilings, Discrete Comput. Geom. 20 (1998), 265-279.

60. P. J. Steinhardt and H.-C. Jeong, A simpler approach to Penrose tiling with implications for quasicrystal formation, Nature 382 (1996), 431-433.

Received February 14, 1997, and in revised form February 14, 1998, February 19, 1998, and March 5, 1998. 